\title{
Asymptotic Properties of the Solutions of Linear and Nonlinear Spin Field Equations in Minkowski Space
}

\author{
Wei-Tong Shu ${ }^{\star}$ \\ Institute for Advanced Study, Princeton, NJ 08540, USA \\ Received October 15, 1990; in revised form December 15, 1990
}

\begin{abstract}
In this paper I will first derive, based on energy estimations and geometric invariance, the asymptotic behavior of solutions of linear spin field equations in Minkowski space. It generalizes the result in [3] where it was proved for the spin-1 and spin-2 cases. The techniques are then applied to Yang-Mills equations, the result improves the previous one in [1] by allowing the initial data to have charge, dipole and quadrupole moments. The Lie derivative operator for spinors and some properties will be also discussed; they can be used to simplify some algebraic calculations of [4].
\end{abstract}

\section{Introduction}

There have been a lot of works which use energy estimates together with the geometric invariance to prove the global existence of a small amplitude solution of nonlinear hyperbolic equations. The highlight is the recent work of D. Christodoulou and S. Klainerman who proved in [4] the stability of Minkowski space; for the simpler case of nonlinear wave equations, see [8]. The most important part of this type of work is to derive a good decay estimate through energy estimations for the solutions of the linearized equations.

In this paper, I will study the asymptotic behavior for the solutions of spin- $n / 2$ equations in Minkowski space $\mathbf{R}^{1+3}$. The special cases of the spin-1/2, spin-1 and spin-2 are respectively the Dirac equations, Maxwell equations and the linearized Einstein equations in a vacuum. Recently, in [3], the asymptotic properties were studied by using energy estimates for the spin- 1 and spin-2 equations without referring to spinors. In this paper I will generalize them to the arbitrary spin case. This result was first obtained by R. Penrose who used the conformal transformation

* This research is partially supported by a grant from NSF under DMS-8610730 
from Minkowski space to the Einstein cylinder $\mathbf{R} \times S^{3}$, see [11,12]. Application of this method to the Yang-Mills equations was done in [1] to prove the global existence of small amplitude solutions when the initial data do not have charge, dipole or quadrupole moments. The decay properties were also obtained in this case. By using the energy estimation, we will prove the similar result to include the case when the initial data has charge, dipole and quadrupole moments. The global existence will also be obtained in this way; it is very easy once we have the necessary estimates.

Apart from the results, another important thing in this paper is the Lie derivative operator for spinors. Some very nice properties of this operator will also be discussed. In [4], a modified Lie derivative operator for the Weyl tensors and Weyl currents was also defined, the operator I defined in this paper agrees with that in [4], but it is much more natural in this spinor formulation, and the properties are much easier to prove in this form. These properties can be used to simplify some calculations in [4] (Part II). Because of this application, most of the expositions in this paper will be done on arbitrary Lorentz manifolds, although the final result will only be proved on Minkowski space.

Without being in their most precise forms, the following two theorems are the main results of this paper,

Theorem 1. For the solution $\psi_{A B}$... of linear spin- $n / 2$ equations in Minkowski space $\mathbf{R}^{1+3}$, we have the following uniform $L^{\infty}$ estimate when $t>0$ (similarly for $t<0$ ),

$$
|\psi(t, x)| \leqq C \tau_{+}^{-1} \tau_{-}^{-n+1 / 2}\|\psi(0, x)\|_{H_{2, n-1}},
$$

where $C$ is a constant independent of the solutions,

$$
\tau_{+}^{2}=1+(t+r)^{2}, \quad \tau_{-}^{2}=1+(t-r)^{2},
$$

and $H_{s, \delta}$ is the weighted Sobolev space whose norm is

$$
\|\psi(x)\|_{\boldsymbol{H}_{s, \delta}}^{2}=\sum_{k \leqq s} \int_{\mathbf{R}^{3}}\left(1+r^{2}\right)^{k+\delta}\left|\nabla^{k} \psi(x)\right|^{2} .
$$

The more precise form of this theorem is the so-called "peeling off" properties of the solutions along the null directions.

Theorem 2. Let $\Psi_{A B}$ be the curvature spinor of the Yang-Mills field. Suppose that the initial data are smooth, satisfy the constraint equations and the following smallness condition:

$$
\|\Psi(0, x)\|_{H_{2,1}^{g}} \leqq \varepsilon_{0},
$$

where $\varepsilon_{0}$ is a small constant; the superscript $g$ on the $H_{2,1}$ norm means that it is the gauge covariant norm, that is, we replace the usual derivative by the gauge covariant derivative in the definition of (3), the inner product of the Lie algebra is taken to be the Killing product $(\cdot, \cdot)$ which is assumed to be positive. Then there exists a unique global solution of Yang-Mills equations in Minkowski space; moreover it has the following decay estimate, when $t>0$,

$$
|\Psi(t, x)| \leqq C \tau_{+}^{-1} \tau_{-}^{-3 / 2}\|\Psi(0, x)\|_{H_{2,1}^{g}} .
$$

The result in Theorem 2 still does not allow the initial data to have charge, that is if the curvature only decays like $r^{-2}$ at $t=0$. But without too much more 
difficulty, I will also prove that for some initial data with charge the estimate (5) is still true inside a fixed light cone, i.e. $r \leqq t+C$; outside the light cone we will only have the following estimate:

$$
|\Psi(t, x)| \leqq C \tau_{+}^{-1} \tau_{-}^{-1}\|\Psi(0, x)\|_{H_{2,1}^{g}} .
$$

The proof of Theorem 2 is based on the à priori estimate of some weighted Sobolev norms. When the group $G$ is non-Abelian, as we begin to differentiate the Yang-Mills equations, we have to estimate some integrals over a region in the space time of the error terms generated from the commutator of the gauge covariant derivative operators. To estimate this integral under the à priori assumption that the curvature spinor $\Psi_{A B}$ decays like the solution of linear spin-1 equations, usually, we split the region into a family of space like hypersurfaces $\Sigma_{t}, t$ is a time function. Then we do some manipulations such as the Hölder inequality on the integrals over $\Sigma_{t}$, and hope to come up with something which is à priori integrable for $t \in(0, \infty)$. For our problem, we encounter serious difficulties in this approach. Technically, this is because we cannot make full use of the à priori assumptions that the components of curvature decay differently along the null directions. The idea is that we can split the space time region into a family of light cones $C_{u}, u$ is an optical function, and perform the similar procedure as before, then integrate along the parameter $u$. This small trick seems to be just a pure technicality, but I believe it is very important in studying problems related to the radiation of solutions of hyperbolic equations because radiation waves propagate along null geodesics, while the concept of space and time is just purely a matter of intuitive convenience.

To illustrate, let's look at how the methods differ in estimating one of the error terms (the notations are given in the subsequent sections):

$$
\begin{aligned}
& \int_{0<t<\infty} \tau_{+}^{2} r\left|\hat{\mathscr{F}}_{\mathcal{O}} \Psi_{1}\left\|\Psi_{-1}\right\| \Psi_{1}\right| \\
& \leqq \int_{0}^{\infty} d t \sup _{\Sigma_{t}}\left(r\left|\Psi_{-1}\right|\right)\left(\int_{\Sigma_{t}} \tau_{+}^{2}\left|\hat{\mathscr{P}}_{\mathcal{O}} \Psi_{1}\right|^{2}\right)^{1 / 2}\left(\int_{\Sigma_{t}} \tau_{+}^{2}\left|\Psi_{1}\right|^{2}\right)^{1 / 2} .
\end{aligned}
$$

The à priori assumption

$$
\left|\Psi_{-1}\right| \leqq C \tau_{+}^{-1} \tau_{-}^{-3 / 2}
$$

implies $\sup \left(r\left|\Psi_{-1}\right|\right) \leqq C$, but this is terrible because the integral in time is not convergent.

Now if we use the family of outgoing light cone $C_{u}=\{t-r=u\}$, the estimate then proceeds as follows:

$$
\begin{aligned}
& \int_{0<t<\infty} \tau_{+}^{2} r\left|\hat{\mathscr{W}}_{\mathcal{O}} \Psi_{1}\left\|\Psi_{-1}\right\| \Psi_{1}\right| \\
& \leqq \int_{-\infty}^{\infty} d u \sup _{C_{u}}\left(r\left|\Psi_{-1}\right|\right)\left(\int_{C_{u}} \tau_{+}^{2}\left|\hat{\mathscr{P}}_{\mathcal{O}} \Psi_{1}\right|^{2}\right)^{1 / 2}\left(\int_{C_{u}} \tau_{+}^{2}\left|\Psi_{1}\right|^{2}\right)^{1 / 2} .
\end{aligned}
$$

The à priori assumption implies $\sup _{C_{u}}\left(r\left|\Psi_{-1}\right|\right) \leqq C /(1+|u|)^{3 / 2}$, which is integrable for $u \in(-\infty, \infty)$. This is good!

The paper is organized as follows: in Sect. 2, I will first review the notations of two component spinors; in Sect. 3, I will discuss the Lie derivative operators; 
Sects. 4 and 5 respectively give the decay estimates for the solutions of spin equations and Yang-Mills equations.

\section{Some Notations about Spinors}

We will use throughout this paper the notations of the two component spinors. In this section I will give a quick review of some of these; more details can be found in [12].

$\left(M^{1+3}, g_{\mu v}\right)$ is a Lorentz manifold, that is $g_{\mu v}$ has signature $(1,-1,-1,-1)$, the Greek letters run from 0 to 3 . If we fixed a time function $t$ on $M$, then choose $x^{i}$ to be the coordinate of $\Sigma_{0}=\{t=0\}$, they are also the coordinates of each time slice $\Sigma_{t}=\{t=$ Const $\}$ by following the time flow; the lower case Latin letters go from 1 to 3 . We will use $D_{\mu}$ to denote the covariant derivative on $M$, and $\nabla_{i}$ the covariant derivative on each time slice.

$\left(V, \varepsilon_{A B}, \sigma_{\mu}^{A B^{\prime}}\right)$ is the spinor structure of $\left(M, g_{\mu v}\right)$, where $V$ is a rank 2 complex vector bundle on $M$; the capital Latin letters such as $A=0,1$ are the index labeling the fibre of $V ; A^{\prime}=0^{\prime}, 1^{\prime}$ is the index labeling the conjugate bundle $\bar{V} ; \varepsilon_{A B}$ is a symplectic product on $V$, i.e. it is nonsingular and $\varepsilon_{A B}=-\varepsilon_{B A} ; \sigma_{\mu}^{A B^{\prime}}=\overline{\sigma_{\mu}^{B A^{\prime}}}$ is a Hermitian spinor valued one form with the following property:

$$
\sigma_{\mu A A^{\prime}} \sigma_{v}^{A A^{\prime}}=g_{\mu v}, \quad \sigma_{\mu A A^{\prime}} \sigma_{B B^{\prime}}^{\mu}=\varepsilon_{A B} \varepsilon_{A^{\prime} B^{\prime}}
$$

and for any future-directed time-like vector field $T, \sigma_{\mu}^{A A^{\prime}} T^{\mu}$ is positive definite.

We use the $\varepsilon$ 's to raise and lower the indices of spinors as follows:

$$
\psi^{A}=\varepsilon^{A B} \psi_{B}, \quad \psi_{A}=\psi^{B} \varepsilon_{B A} .
$$

The following map defines an isometry from the tangent bundle $T M$ to the Hermitian subbundle of $V \otimes \bar{V}$ :

$$
X^{\mu} \rightarrow X^{A A^{\prime}}=X^{\mu} \sigma_{\mu}^{A A^{\prime}} .
$$

In a similar way, any tensor is identified as a spinor; denote $D_{A A^{\prime}}=\sigma_{A A^{\prime}}^{\mu} D_{\mu}$ to be the covariant derivative operator for spinors.

Definition 1. If spinor $\psi_{\underbrace{A B \ldots}_{\text {times }}}$ is totally symmetric with respect to the indices $(A B \ldots)$, then it is called a spin- $n / 2$ field; and

$$
D^{A A^{\prime}} \psi_{A B \ldots}=0
$$

is called the (massless) spin- $n / 2$ field equation.

As an example I will recall how to write the usual Yang-Mills equations as the gauge covariant spin- 1 equations. Let $G$ be a compact Lie group, $\mathscr{G}$ be its Lie algebra. A gauge field is given by its gauge potential $\phi_{\mu}$ which is a $\mathscr{G}$ valued one form. Denote $\mathbf{D}_{\mu}=D_{\mu}+\left[\phi_{\mu}, \cdot\right]$ the gauge covariant derivative. Let $F_{\mu v}=\partial_{\mu} \phi_{v}-$ $\partial_{\nu} \phi_{\mu}+\left[\phi_{\mu}, \phi_{v}\right]$, it is called the curvature of $\phi_{\mu}$.

$F_{\mu \nu}$ satisfies the following Bianchi identity:

$$
\mathbf{D}^{\mu} F_{\mu \nu}^{*}=D^{\mu} F_{\mu \nu}^{*}+\left[\phi^{\mu}, F_{\mu \nu}^{*}\right]=0,
$$

where $F_{\mu \nu}^{*}=\frac{1}{2} \varepsilon_{\mu \nu \alpha \beta} F^{\alpha \beta}, \varepsilon_{\alpha \beta \mu \nu}$ is the volume form of $\left(M^{1+3}, g_{\mu \nu}\right)$. 
The gauge field is said to satisfy the Yang-Mills equations if

$$
\mathbf{D}^{\mu} F_{\mu \nu}=D^{\mu} F_{\mu \nu}+\left[\phi^{\mu}, F_{\mu \nu}\right]=0 \text {. }
$$

Let

$$
\Psi_{A B}=\frac{1}{2} F_{A A^{\prime} B} A^{\prime},
$$

then

$$
\begin{aligned}
& F_{\mu \nu}=\Psi_{A B} \varepsilon_{A^{\prime} B^{\prime}}+\bar{\Psi}_{A^{\prime} B^{\prime}} \varepsilon_{A B}, \\
& F_{\mu \nu}^{*}=-i \Psi_{A B^{\prime} \varepsilon_{A^{\prime} B^{\prime}}}+i \bar{\Psi}_{A^{\prime} B^{\prime}} \varepsilon_{A B} .
\end{aligned}
$$

Therefore the Yang-Mills equations are equivalent to the gauge covariant spin-1 equations, namely

$$
\mathrm{D}^{A A^{\prime}} \Psi_{A B}=D^{A A^{\prime}} \Psi_{A B}+\left[\phi^{A A^{\prime}}, \Psi_{A B}\right]=0 .
$$

Lemma 1. For any spin- $n / 2$ field $\psi_{A B \ldots}$, define a n-tensor

$$
Q_{\mu v \ldots}(\psi)=\psi_{A B \ldots} \bar{\psi}_{A^{\prime} B^{\prime} \ldots},
$$

it is called the energy tensor of the spin field $\psi_{A B \ldots}$.

1. $Q_{\mu \nu \ldots}$ is totally symmetric and traceless with respect to any pair of indices.

2. If $\dddot{X}^{\mu}, Y^{\mu}, \ldots$ are future-directed nonspace-like vectors, then,

$$
Q(X, Y, \ldots)=Q_{\mu \nu \ldots} X^{\mu} Y^{\nu} \ldots \geqq 0 .
$$

3. If $\psi_{A B \ldots}$ satisfies the spin equation (10), then

$$
D^{\mu} Q_{\mu \nu \ldots}=0 \text {. }
$$

Remark that for the spin-1 field $\psi_{A B}, Q_{\mu v}(\psi)$ is the energy-momentum tensor; for the spin-2 field $\psi_{A B C D}, Q_{\mu \nu \alpha \beta}(\psi)$ is the Bell-Robinson tensor.

Next, we will discuss the so-called Newman-Penrose formalism in general relativity. This formalism is based on the following simple fact:

A future-directed real vector $X^{\mu}$ is null $\Leftrightarrow$ there is a spinor $\psi^{A}$ such that $X^{A A^{\prime}}=\psi^{A} \bar{\psi}^{A^{\prime}}$; such a spinor is determined by the direction of $X^{\mu}$ up to the scaling $\psi^{A} \rightarrow \lambda \psi^{A}, \lambda$ is a nonzero complex number.

In this paper, we will foliate a space time by two families of null hypersurfaces $C_{u}$ and $C_{v}^{\prime}$, where $u, v$ are parameters which are called optical functions. Let $S_{u, v}=C_{u} \cap C_{v}^{\prime}$; they are two dimensional space-like surfaces. Let $l$ be the null generator of $C_{u}$, and $l^{\prime}$ be the null generator of $C_{v}^{\prime}$, such that $l_{\mu}^{\prime} l^{\mu}=1$.

Choose the spin frame $\left\{\xi^{A}, \eta^{A}\right\}$ such that

$$
\xi_{A} \eta^{A}=1, \quad l^{\mu}=\xi^{A} \bar{\xi}^{A^{\prime}}, \quad l^{\mu}=\eta^{A} \bar{\eta}^{A^{\prime}},
$$

then $\left\{\xi^{A}, \eta^{A}\right\}$ is determined by $C_{u}$ and $C_{v}^{\prime}$ up to the following spin transformation:

$$
\left(\xi^{A}, \eta^{A}\right) \rightarrow\left(\lambda \xi^{A}, \lambda^{-1} \eta^{A}\right)
$$

where $\lambda$ is any nonzero complex number.

Definition 2. Let $\psi$ be some quantity which depends on the choice of spin frame $\left\{\xi^{A}, \eta^{A}\right\}$, if under the above spin transformation (19)

$$
\psi \rightarrow \lambda^{p} \bar{\lambda}^{q} \psi
$$

then $\psi$ is called $a(p, q)$ weighted quantity. 
Examples.

1. For any spin- $n / 2$ field $\psi_{A B \ldots}$, define

$$
\psi_{k}=\underbrace{\xi^{A} \cdots \xi^{B}}_{n / 2+k \text { times }} \underbrace{\eta^{C} \cdots \eta_{\text {times }}^{D}} \psi_{A B C D \ldots}
$$

where $k=-n / 2,-n / 2+1, \ldots, n / 2$, then $\psi_{k}$ is a $(2 k, 0)$ weighted scalar. I remark that for the spin-1 and spin-2 field, this decomposition is the null decomposition for the electric magnetic field and the Weyl field defined in $[3,4]$.

2. For a spinor $J_{A^{\prime}} \underbrace{A B \ldots}_{n-1 \text { tumes }}$, if $J_{A^{\prime} A B \ldots}=J_{A^{\prime}(A B \ldots)}$, then it is called a spin- $n / 2$ current. For $k=-n / 2, \ldots, n / 2-1$, let

$$
\begin{aligned}
J_{k} & =\bar{\xi}^{A^{\prime}} \underbrace{\xi^{A} \cdots \xi^{B} \xi^{B}}_{n / 2+k \text { times }} \underbrace{\eta^{C} \cdots \eta^{D}} J_{A^{\prime} A B C D} \ldots \\
J_{-k-1}^{\prime} & =i^{n-2} \bar{\eta}^{A^{\prime}} \underbrace{\xi^{A} \cdots \xi^{B}}_{n / 2+k \text { times }} \underbrace{\eta^{C} \cdots \eta^{D}} J_{A^{\prime} A B C D \ldots} \ldots
\end{aligned}
$$

then $J_{k}$ is $(2 k+1,1)$ weighted, $J_{k}^{\prime}$ is $(-2 k-1,-1)$ weighted.

Definition 3. For any given spin frame $\left\{\xi^{A}, \eta^{A}\right\}$, let

$$
\begin{aligned}
D & =l^{\mu} D_{\mu}, & D^{\prime} & =l^{\mu} D_{\mu}, \\
\delta & =m^{\mu} D_{\mu}, & \delta^{\prime} & =m^{\prime \mu} D_{\mu},
\end{aligned}
$$

where $m^{\mu}=\xi^{A} \bar{\eta}^{A^{\prime}}, m^{\prime \mu}=\eta^{A} \bar{\xi}^{A^{\prime}}=\bar{m}^{\mu}$ are tangent to $S_{u, v}$. Suppose

$$
\begin{aligned}
D \xi^{A} & =\varepsilon \xi^{A}-\kappa \eta^{A}, & D^{\prime} \eta^{A} & =\varepsilon^{\prime} \eta^{A}-\kappa^{\prime} \xi^{A}, \\
\delta^{\prime} \xi^{A} & =\alpha \xi^{A}-\rho \eta^{A}, & \delta \eta^{A} & =\alpha^{\prime} \eta^{A}-\rho^{\prime} \xi^{A}, \\
\delta \xi^{A} & =\beta \xi^{A}-\sigma \eta^{A}, & \delta^{\prime} \eta^{A} & =\beta^{\prime} \eta^{A}-\sigma^{\prime} \xi^{A}, \\
D^{\prime} \xi^{A} & =\gamma \xi^{A}-\tau \eta^{A}, & D \eta^{A} & =\gamma^{\prime} \eta^{A}-\tau^{\prime} \xi^{A},
\end{aligned}
$$

then $\varepsilon, \alpha, \ldots$, etc. are called the Ricci coefficients of $\left\{\xi^{A}, \eta^{A}\right\}$.

In fact we have the following relations among the Ricci coefficients:

$$
\begin{aligned}
\varepsilon+\gamma^{\prime}=0, & \varepsilon^{\prime}+\gamma=0, \\
\alpha+\beta^{\prime}=0, & \alpha^{\prime}+\beta=0 . \\
\kappa=0, & \kappa^{\prime}=0, \\
\rho=\bar{\rho}, & \rho^{\prime}=\bar{\rho}^{\prime},
\end{aligned}
$$

where we differentiate $\xi_{A} \eta^{A}=1$ to obtain (26), and (27) is a result of the Frobenius Theorem for the submanifolds $C_{u}$ and $C_{v}^{\prime}$.

The above notations are greatly simplified by using the primes. The reason for this prime operations is that if we let

$$
\xi^{A}=i \eta^{A}, \quad \eta^{\prime A}=i \xi^{A},
$$

then $\left\{\xi^{\prime A}, \eta^{\prime A}\right\}$ is another spin frame. Under this spin transformation, we interchange the two generators $l$ and $l^{\prime}$. A quantity associated with the prime frame is the 
prime of the same quantity associated with the original frame. Obviously, $\psi_{k}^{\prime}=i^{n} \psi_{-k}$. From now on we will always omit writing down the prime quantities.

The following three remarks are obvious to show:

1. If $\psi$ is $(p, q)$ weighted, then $\psi^{\prime}$ is $(-p,-q)$ weighted, and $\bar{\psi}$ is $(q, p)$ weighted.

2. The Ricci coefficients $\kappa, \rho, \sigma, \tau$ are weighted scalars whose weights are $(3,1),(1,1),(3,-1),(1,-1)$ respectively.

3. Scalars $\varepsilon, \alpha, \beta, \gamma$ are not weighted, nor are the derivative operators $D$ and $\delta$, but if we define the following two operators for any $(p, q)$ weighted scalar $\psi$,

$$
\begin{aligned}
\wp \psi & =(D-p \varepsilon-q \bar{\varepsilon}) \psi, \\
\partial \psi & =(\delta-p \beta-q \bar{\alpha}) \psi,
\end{aligned}
$$

then $\wp, \varnothing$ are weighted derivative operators with weights $(1,1)$ and $(1,-1)$ respectively, that is $\wp \psi$ and $\phi \psi$ are respectively $(p+1, q+1)$ and $(p+1, q-1)$ weighted scalars.

The weights are very useful to check whether or not the calculations are correct; the prime operation reduces anything we have to do by half.

Lemma 2. If $D^{A A^{\prime}} \psi_{A B \ldots}=J_{B \ldots}^{A^{\prime}}$, then for $k=-n / 2, \ldots, n / 2-1$,

$$
\begin{aligned}
\wp \psi_{k}-\not \partial^{\prime} \psi_{k+1}= & -(n / 2+k) \kappa \psi_{k-1}+(n / 2+k+1) \rho \psi_{k} \\
& -(n / 2-k) \tau^{\prime} \psi_{k+1}+(n / 2-k-1) \sigma^{\prime} \psi_{k+2}-J_{k} .
\end{aligned}
$$

If we do not need to know the exact numerical coefficients on the right-hand side of (29), the equations can immediately be proved just by comparing the weights of both sides. The actual proof is just a straightforward calculation, see [12].

In Minkowski space, we will use the following two optical functions: $u=t-r$, $v=t+r$. Choose the generators of $C_{u}=\{u=$ Const $\}$ and $C_{v}^{\prime}=\{v=$ Const $\}$ to be

$$
l=\frac{1}{\sqrt{2}}\left(\frac{\partial}{\partial t}+\frac{\partial}{\partial r}\right), \quad l^{\prime}=-\frac{1}{\sqrt{2}}\left(\frac{\partial}{\partial t}-\frac{\partial}{\partial r}\right),
$$

then the spin frame $\left\{\xi^{A}, \eta^{A}\right\}$ is determined up to the scaling (19) with $|\lambda|=1$. Hence if $\psi$ is $(p, q)$ weighted, let $s=(p-q) / 2$, then under (19),

$$
\psi \rightarrow \lambda^{2 s} \psi,
$$

$s$ is called the spin weight of $\psi$.

For any spin- $n / 2$ field $\psi_{A B \ldots}$, and any spin- $n / 2$ current $J_{A^{\prime} A B \ldots}$, both $\psi_{k}$ and $J_{k}$ have spin weight $k$. This is why we arranged the definition as in (21), (22).

One can easily compute the spin coefficients of Minkowski space under this spin frame to find

$$
\begin{array}{lll}
\varepsilon=0, & \varepsilon^{\prime}=0, \\
\kappa=0, & \kappa^{\prime}=0, \\
\rho=-\frac{1}{\sqrt{2}}, & \rho^{\prime}=-\frac{1}{\sqrt{2} r}, \\
\sigma=0, & & \sigma^{\prime}=0, \\
\tau=0, & \tau^{\prime}=0 .
\end{array}
$$


Therefore if $D^{A A^{\prime}} \psi_{A A \ldots}=J_{B \ldots}^{A^{\prime}}$, then

$$
\begin{gathered}
\left(D+(n / 2+k+1) \frac{1}{\sqrt{2} r}\right) \psi_{k}-\not \partial^{\prime} \psi_{k+1}=-J_{k}, \\
\left(D^{\prime}-(n / 2-k+1) \frac{1}{\sqrt{2} r}\right) \psi_{-k}-\not \partial \psi_{-k-1}=J_{-k}^{\prime},
\end{gathered}
$$

where $D=\left(\partial_{t}+\partial_{r}\right) / \sqrt{2}, D^{\prime}=\left(\partial_{t}-\partial_{r}\right) / \sqrt{2} ; \partial$ can be identified as the Dirac operator on $S_{t, r}=\{t=$ const, $r=$ const $\} ; \psi_{k}, J_{k}$ and $J_{k}^{\prime}$ are defined in (21)-(23).

\section{Lie Derivatives of Spinors}

There is a conceptual difficulty in defining the Lie derivative for spinors. Recall that for any one from $\phi_{\mu}$, the Lie derivative $\mathscr{L}_{X} \phi_{\mu}$ is defined to be $\left.\frac{d}{d t}\right|_{t=0} f_{t}^{*} \phi_{\mu}$, where $f_{t}$ is the one parameter family of diffeomorphisms which generate the vector field $X$ such that $f_{0}$ is the identity map of $M$. Unless $X$ is conformal Killing, if the one form $\phi_{\mu}$ is null, in general $f_{t}^{*} \phi_{\mu}$ is not null any more. But as we have discussed in Sect. 2, a spinor naturally defines a null object through the spin structure. Therefore it is not possible to define the Lie derivative of a spinor for any vector field $X$ such that it gives us the usual Lie derivative for tensors when it is restricted to tensors; or equivalently, it is not possible to define a Lie derivative operator for spinors such that it is compatible with the spin structure and the usual Lie derivative operator for tensors. This is because the definition of the spinor structure in Sect. 2 is a structure on a manifold with a Lorentz metric, while the usual definition of Lie derivative operator for tensors has nothing to do with the metric.

To define the Lie derivative for spinors, we will forget the usual definition of the Lie derivative for tensors, instead we require that it is compatible with the spin structure, that is

$$
\begin{gathered}
\mathscr{L}_{X} \varepsilon_{A B}=0, \\
\mathscr{L}_{X} \sigma_{\mu}^{A B^{\prime}}=0 .
\end{gathered}
$$

This leads us to the following definition:

Definition 4. Given any tangent vector field $X$, define the Lie derivative of spinor $\psi_{A} b y$

$$
\mathscr{L}_{X} \psi_{A}=X^{\mu} D_{\mu} \psi_{A}+h_{A}^{B} \psi_{B}
$$

where

$$
h_{A B}=\frac{1}{2} D_{A^{\prime}(A} X_{B)}^{A^{\prime}}
$$

For other types of spinors, the corresponding Lie derivatives are defined in the usual 
fashion from (34). For example,

$$
\begin{aligned}
\mathscr{L}_{X} \psi^{A} & =X^{\mu} D_{\mu} \psi^{A}-\psi^{B} h_{B}^{A}, \\
\mathscr{L}_{X} \psi_{A^{\prime}} & =X^{\mu} D_{\mu} \psi_{A^{\prime}}+\bar{h}_{A^{\prime}}^{B^{\prime}} \psi_{B^{\prime}}, \\
\mathscr{L}_{X} \psi_{A B} & =X^{\mu} D_{\mu} \psi_{A B}+h_{A}^{C} \psi_{C B}+h_{B}^{C} \psi_{A C} .
\end{aligned}
$$

Remarks. 1. Under this definition, the Lie derivative for an one form $\varphi_{\mu}$ becomes

$$
\mathscr{L}_{X} \varphi_{\mu}=X^{v} D_{v} \varphi_{\mu}+\frac{1}{2} \varphi_{v}\left(D_{\mu} X^{v}-D^{v} X_{\mu}\right)
$$

This is because by the definition of $h_{A B}$, we have

$$
h_{A B} \varepsilon_{A^{\prime} B^{\prime}}+\bar{h}_{A^{\prime} B^{\prime}} \varepsilon_{A B}=\frac{1}{2}\left(D_{\mu} X_{v}-D_{v} X_{\mu}\right) \text {. }
$$

Therefore this new Lie derivative operator agrees with the old one for tensors if and only if $X$ is a Killing vector field.

2. In $[3,4]$, in order to preserve the traceless property for a spin-2 field (in tensor form), they had to modify the definition of Lie derivative. Up to a correction term with $\operatorname{tr} \pi$, the definition given there agrees with the one $I$ gave here for the spin-2 fields (see (40), (41) for the definition of $\hat{\mathscr{L}}_{X}$ ). The correction involving $\operatorname{tr} \pi$ can also be removed if we do not require $\mathscr{L}_{X} \varepsilon_{A B}=0$ in the definition.

Let's first look at the relation between this Lie derivative operator and the Newman-Penrose formalism in the previous section. by

For any spin frame $\left\{\xi^{A}, \eta^{A}\right\}$, define scalar functions $z(X), w(X)$ and $z^{\prime}(X), w^{\prime}(X)$

$$
\begin{aligned}
& \mathscr{L}_{X} \xi^{A}=z(X) \xi^{A}-w(X) \eta^{A} \\
& \mathscr{L}_{X} \eta^{A}=z^{\prime}(X) \eta^{A}-w^{\prime}(X) \xi^{A} .
\end{aligned}
$$

It is easy to see that $w(X)$ and $w^{\prime}(X)$ are weighted scalars whose weights are $(2,0)$ and $(-2,0)$ respectively. Since we normalize the spin frame $\left\{\xi^{A}, \eta^{A}\right\}$ such that $\xi_{A} \eta^{A}=1$, we know $z^{\prime}(X)=-z(X)$. Like the Ricci coefficients $\varepsilon, \alpha, \beta$ and $\gamma$, the scalar $z(X)$ is not a weighted quantity, neither is the Lie derivative operator $\mathscr{L}_{X}$, but if we define

$$
\mathscr{L}_{X} \psi=\mathscr{L}_{X} \psi-p z(X) \psi-q \bar{z}(X) \psi
$$

for any $(p, q)$ weighted quantity $\psi$, then $\mathscr{L}_{X}$ is a $(0,0)$ weighted derivative operator.

Lemma 3. For any spin- $n / 2$ field $\psi_{A B \ldots}$, let $\psi_{k}\left(\mathscr{L}_{X} \psi\right)$ denote the components of $\mathscr{L}_{X} \psi_{A B C D \ldots}$ (cf. (21)), then

$$
\psi_{k}\left(\mathscr{L}_{X} \psi\right)=\mathscr{L}_{X} \psi_{k}+(n / 2+k) w(X) \psi_{k-1}+(n / 2-k) w^{\prime}(X) \psi_{k+1},
$$

where $k=-n / 2, \ldots, n / 2$.

Proof.

$$
\begin{aligned}
\psi_{k}\left(\mathscr{L}_{X} \psi\right)= & \underbrace{\xi^{A} \cdots \xi^{A}-k \text { times }}_{n / 2+k \text { times }} \underbrace{\eta^{C} \cdots \eta^{D}} \mathscr{L}_{X} \psi_{A B C D \ldots} \\
= & \mathscr{L}_{X} \psi_{k}-(n / 2+k)\left(\mathscr{L}_{X} \xi^{A}\right) \underbrace{\xi^{B} \cdots \xi^{C}}_{n / 2+k-1 \text { times }} \underbrace{\eta^{D} \cdots \eta^{E}}_{n / 2-k \text { times }} \psi_{A B \ldots} \\
& -(n / 2-k)\left(\mathscr{L}_{X} \eta^{A}\right) \underbrace{\xi^{B} \cdots \xi^{C}}_{n / 2+k \text { times }} \underbrace{\eta^{D} \cdots \eta^{E}}_{n-k-1 \text { times }} \psi_{A B \ldots} .
\end{aligned}
$$


Because the right-hand side has to be weighted,

$$
\begin{aligned}
\psi_{k}\left(\mathscr{L}_{X} \psi\right)= & \mathscr{L}_{X} \psi_{k}-(n / 2+k)(\mathscr{L}_{X}^{\left.\xi^{A}\right)} \underbrace{\xi^{B} \cdots \xi^{C}}_{n / 2+k-1 \text { times }} \underbrace{\eta^{D} \cdots \eta^{E}} \psi_{A B \ldots i m e s} \\
& -(n / 2-k)\left(\mathscr{L}_{X} \eta^{A}\right) \underbrace{\xi^{B} \cdots \xi^{C}}_{n / 2+k \text { times }} \underbrace{\eta^{D} \cdots \eta^{E}} \psi_{A B \cdots} \\
= & \mathscr{L}_{X} \psi_{k}+(n / 2+k) w(X) \psi_{k-1}+(n / 2-k) w^{\prime}(X) \psi_{k+1} .
\end{aligned}
$$

$w(X)$ and $w^{\prime}(X)$ can be calculated explicitly as follows,

Lemma 4. For any vector field $X$, denote ${ }^{(X)} \pi_{\mu \nu}=\nabla_{\mu} X_{v}+\nabla_{v} X_{\mu}$; it is called the deformation tensor of $X$, let ${ }^{(X)} \hat{\pi}$ be the traceless part of it.

1. If $X$ is tangent to the two surfaces $S_{u, v}$, then

$$
w(X)=-\frac{1}{2}^{(X)} \hat{\pi}_{m l}, \quad w^{\prime}(X)=-\frac{1}{2}^{(X)} \hat{\pi}_{m^{\prime} l^{\prime}} .
$$

2. Suppose the distribution spanned by the generators $l$ and $l^{\prime}$ is integrable. If $X$ is perpendicular to $S_{u, v}$, then

$$
w(X)=\frac{1}{2}^{(X)} \hat{\pi}_{m l}, \quad w^{\prime}(X)=\frac{1}{2}^{(X)} \hat{\pi}_{m^{\prime} l^{\prime}} .
$$

Proof. For any vector field $X=f l+f^{\prime} l^{\prime}+g m+g^{\prime} m^{\prime}$, then

$$
\begin{aligned}
w(X)= & -\xi_{A} \mathscr{L}_{X} \xi^{A}=\xi^{A} \mathscr{L}_{X} \xi_{A}=\xi^{A} X^{\mu} D_{\mu} \xi_{A}-h_{A B} \xi^{A} \xi^{B} \\
= & \xi^{A} X^{\mu} D_{\mu} \xi_{A}+\frac{1}{2}\left(l^{\mu} \partial X_{\mu}-m^{\mu} \wp X_{\mu}\right) \\
= & \xi^{A}\left(f D \xi_{A}+f^{\prime} D^{\prime} \xi_{A}+g \delta \xi_{A}+g^{\prime} \delta^{\prime} \xi_{A}\right) \\
& +\frac{1}{2} l^{\mu} \not \partial\left(f l_{\mu}+f^{\prime} l_{\mu}^{\prime}+g m_{\mu}+g^{\prime} m_{\mu}^{\prime}\right) \\
& -\frac{1}{2} m \mu \wp\left(l_{\mu}+f^{\prime} l_{\mu}^{\prime}+g m_{\mu}+g^{\prime} m_{\mu}^{\prime}\right) \\
= & \frac{1}{2}\left\{\kappa f+\left(\not+2 \tau-\bar{\tau}^{\prime}\right) f^{\prime}+\sigma g+(\wp+2 \rho-\bar{p}) g^{\prime}\right\} .
\end{aligned}
$$

The validity of the above identity can easily be seen by comparing the weights on both sides. Compute

$$
\begin{aligned}
\hat{\pi}_{m l} & =l^{\mu} \partial X_{\mu}+m^{\mu} \wp X_{\mu} \\
& =\kappa f+\left(\not \partial+\bar{\tau}^{\prime}\right) f^{\prime}-\sigma g-(\wp+\bar{\rho}) g^{\prime} .
\end{aligned}
$$

If $X$ is tangent to the two surfaces $S_{u, v}$, i.e. $f=0, f^{\prime}=0$, since $\rho=\bar{\rho}$, we have,

$$
w(X)=-\frac{1}{2} \hat{\pi}_{m l}
$$

If $X$ is prependicular to $S_{u, v}$, i.e. $g=0, g^{\prime}=0$, then

$$
w(X)=\frac{1}{2} \hat{\pi}_{m l}+\left(\tau-\bar{\tau}^{\prime}\right) f^{\prime} .
$$

From the Frobenius Theorem, that the distribution spanned by the generators $l$ and $l^{\prime}$ is integrable implies $\tau=\bar{\tau}^{\prime}$, thus

$$
w(X)=\frac{1}{2} \hat{\pi}_{m l} .
$$

In particular, if $X$ is a conformal Killing vector field such that it is either tangent or perpendicular to the two surfaces $S_{u, v}$, for each $u$ and $v$, then $w(X)=0$. Therefore we have the following corollary on the Minkowski space, 
Corollary. If $X$ is the Killing vector field of time translation or spatial rotations

$$
T=\frac{\partial}{\partial t}, \quad \Omega_{i j}=x_{i} \frac{\partial}{\partial x^{j}}-x_{j} \frac{\partial}{\partial x^{i}},
$$

or the conformal Killing vector fields of scaling or inversion

$$
S=t \frac{\partial}{\partial t}+r \frac{\partial}{\partial r}, \quad \bar{K}_{0}=\left(1+t^{2}+r^{2}\right) \frac{\partial}{\partial t}+2 \operatorname{tr} \frac{\partial}{\partial r},
$$

then $w(X)=0, w^{\prime}(X)=0$. Therefore $\psi_{k}\left(\mathscr{L}_{X} \psi\right)=\mathscr{L}_{X} \psi_{k}$.

Finally we want to examine how the spin equations change after taking Lie derivatives.

Lemma 5. For any spin-n/2 field $\psi_{A B \ldots}$ and any spin- $n / 2$ current $J_{A^{\prime} A B \ldots}$, define

$$
\begin{gathered}
\hat{\mathscr{L}}_{X} \psi_{A B \ldots}=\left(\mathscr{L}_{X}+\frac{n+2}{16} \operatorname{tr} \pi\right) \psi_{A B \ldots}, \\
\hat{\mathscr{L}}_{X} J_{A^{\prime} A B \ldots}=\left(\mathscr{L}_{X}+\frac{n+4}{16} \operatorname{tr} \pi\right) J_{A^{\prime} A B \ldots}
\end{gathered}
$$

for any vector field $X$, then

$$
\begin{aligned}
D^{A A^{\prime}} \hat{\mathscr{L}}_{X} \psi_{A B \ldots}= & \hat{\mathscr{L}}_{X} D^{A A^{\prime}} \psi_{A B \ldots} \\
& +\frac{1}{2} \hat{\pi}^{A A^{\prime} C C^{\prime}} D_{C C^{\prime}} \psi_{A B \ldots}+\frac{1}{4} D_{C C^{\prime}} \hat{\pi}^{A A^{\prime} C C^{\prime}} \psi_{A B \ldots} \\
& +\frac{n-1}{4}\left\{\psi_{A C(\ldots} D_{B) C^{\prime}} \hat{\pi}^{A A^{\prime} C C^{\prime}}+C_{C^{\prime}}^{C} \hat{\pi}_{(B}^{A A^{\prime} C^{\prime}} \psi_{\ldots) A C}\right\} \\
:= & J_{B \ldots}^{A^{\prime}}(X, \psi) .
\end{aligned}
$$

We will also denote $J_{A^{\prime} A B \ldots}(X, Y, \psi)=J_{A^{\prime} A B \ldots}\left(Y, \hat{\mathscr{L}}_{X} \psi\right)$.

Proof. In the following proof, I will restrict myself to the case when the underlining space is Minkowski space, but the same lemma holds true as well even in curved space. The same proof will work, we just need to keep track of the terms involving curvatures, but they will vanish eventually; this fact is actually rather remarkable, for a detailed proof see [13].

First, the following identity is just a result of a simple calculation from the definitions,

$$
D_{A A^{\prime}} h_{B C}=\frac{1}{2} D_{B^{\prime}(B} \hat{\pi}_{C) A A^{\prime}}^{B^{\prime}}+\frac{1}{8} \varepsilon_{A(B} D_{C) A^{\prime}} \operatorname{tr} \pi .
$$

The proof of the lemma is through the following calculation,

$$
\begin{aligned}
& D^{A A^{\prime}} \mathscr{L}_{X} \psi_{A B \ldots}=D^{A A^{\prime}}\left\{X^{\mu} D_{\mu} \psi_{A B \ldots}+h_{A}^{C} \psi_{C B \ldots}+(n-1) \psi_{A C(\ldots} h_{B)}^{C}\right\} \\
& =X^{\mu} D_{\mu} D^{A A^{\prime}} \psi_{A B \ldots}+X_{C C^{\prime}}\left[D^{A A^{\prime}}, D^{C C^{\prime}}\right] \psi_{A B \ldots} \\
& +\frac{1}{2} \pi^{A A^{\prime} C C^{\prime}} D_{C C^{\prime}} \psi_{A B \ldots}+\left(h^{A C} \varepsilon^{A^{\prime} C^{\prime}}+\bar{h}^{A^{\prime} C^{\prime}} \varepsilon^{A C}\right) D_{C C^{\prime}} \psi_{A B \ldots} \\
& +h_{A}^{C} D^{A A^{\prime}} \psi_{C B \ldots}+(n-1) h_{(B}^{C} D^{A A^{\prime}} \psi_{\ldots) A C} \\
& +D^{A A^{\prime}} h_{A}^{C} \psi_{C B \ldots}+(n-1) D^{A A^{\prime}} h_{(B}^{C} \psi_{\ldots) A C}
\end{aligned}
$$




$$
\begin{aligned}
& =\mathscr{L}_{X} D^{A A^{\prime}} \psi_{A B \ldots}+\frac{1}{8} \operatorname{tr} \pi D^{A A^{\prime}} \psi_{A B \ldots}+\frac{1}{2} \hat{\pi}^{A A^{\prime} C C^{\prime}} D_{C C^{\prime}} \psi_{A B \ldots} \\
& +D^{A A^{\prime}} h_{A}^{C} \psi_{C B \ldots}+(n-1) D_{A}^{A^{\prime}} h_{C(B} \psi_{\ldots)}^{A C} \\
& =\left(\mathscr{L}_{X}+\frac{1}{8} \operatorname{tr} \pi\right) D^{A A^{\prime}} \psi_{A B \ldots}+\frac{1}{2} \hat{\pi}^{A C A^{\prime} C^{\prime}} D_{C C^{\prime}} \psi_{A B \ldots} \\
& +\left(\frac{1}{4} D_{A B^{\prime}} \hat{\pi}^{A C A^{\prime} B^{\prime}}-\frac{3}{16} D^{C A^{\prime}} \operatorname{tr} \pi\right) \psi_{C B \ldots} \\
& +(n-1)\left[\frac{1}{2} D_{B^{\prime}(B} \hat{\pi}_{A C}^{A^{\prime} B^{\prime}}+\frac{1}{8} D_{C}^{A^{\prime}} \operatorname{tr} \pi \varepsilon_{A(B}\right] \psi_{\ldots)}^{A C} \\
& =\left(\mathscr{L}_{X}+\frac{1}{8} \operatorname{tr} \pi\right) D^{A A^{\prime}} \psi_{A B \ldots} \\
& -\frac{3}{16} D^{A A^{\prime}}\left(\operatorname{tr} \pi \psi_{A B \ldots}\right)+\frac{3}{16} \operatorname{tr} \pi D^{A A^{\prime}} \psi_{A B \ldots} \\
& +\frac{1}{2} \hat{\pi}^{A A^{\prime} C C^{\prime}} D_{C C^{\prime}} \psi_{A B \ldots}+\frac{1}{4} D_{C C^{\prime}}, \hat{\pi}^{A A^{\prime} C C^{\prime}} \psi_{A B \ldots} \\
& +\frac{n-1}{16} D^{A A^{\prime}}\left(\operatorname{tr} \pi \psi_{A B \ldots}\right)+\frac{n-1}{16} \operatorname{tr} \pi D^{A A^{\prime}} \psi_{A B \ldots} \\
& +\frac{n-1}{4} D_{C^{\prime}(B} \hat{\pi}^{A A^{\prime} C C^{\prime}} \psi_{\ldots) A C}+\frac{n-1}{4} D_{C^{\prime}}^{C} \hat{\pi}_{(B}^{A A^{\prime} C^{\prime}} \psi_{\ldots) A C} \\
& =J_{B C \ldots}^{A^{\prime}}(X, \psi)-\frac{n+2}{16} D^{A A^{\prime}}\left(\operatorname{tr} \pi \psi_{A B \ldots}\right) \text {. }
\end{aligned}
$$

\section{Decay Estimates for the Spin-n/2 Equations in Minkowski Space}

In this section, we will prove the following theorem,

Theorem 1. If $\psi_{A B \ldots}$ satisfies the spin-n/2 Eq. (10) in Minkowski space, let

$$
[\psi](T)=\sup _{0<t<T}\left\{\tau_{+}^{n+1 / 2}\left|\psi_{n / 2}\right|+\sum_{k=-n / 2}^{n / 2-1} \tau_{+}^{n / 2+k+1} \tau_{-}^{(n-1) / 2-k}\left|\psi_{k}\right|\right\},
$$

then

$$
[\psi]:=[\psi](\infty) \leqq C\|\psi(0, x)\|_{H_{2, n-1}} .
$$

The above decay estimate is called the "peeling off" property of the spin- $n / 2$ field $\psi_{A B \ldots}$; namely, along the null directions, say along $t=r$, then the $n$ different components $\psi_{k}$ decay as follows:

$$
\left|\psi_{k}\right| \leqq C r^{-n / 2-k-1}, \quad k=-n / 2, \ldots, n / 2-1, \quad\left|\psi_{n / 2}\right| \leqq C r^{-n-1 / 2} .
$$

Remark that $\psi_{k}$ decays one order (i.e. $r^{-1}$ ) better than $\psi_{k-1}$ for $k<n / 2$, but $\psi_{n / 2}$ decays only a half order (i.e. $r^{-1 / 2}$ ) better than $\psi_{n / 2-1}$, while by using the Penrose conformal transformation, one can prove that it is also one order better, i.e. $\left|\psi_{n / 2}\right| \leqq C r^{-n-1}$. Of course the initial data then have to decay faster. In terms of decay at future null infinity, the worst term is $\psi_{-n / 2}$; it decays like $r^{-1}$. This is what reflects the radiation property of the spin- $n / 2$ field equations.

We will use the following notations to denote the different regions in Minkowski space: for $0<T \leqq \infty, u=t-r, v=t+r$, let

$$
V_{T}=\{0<t<T\},
$$


denote the interior and exterior regions by

$$
V_{T}^{i}=\left\{r<\frac{1+t}{3}\right\} \cap V_{T}, \quad V_{T}^{e}=\left\{r>\frac{1+t}{4}\right\} \cap V_{T} .
$$

Let $\Sigma_{t}, C_{u}(T)$ and $C_{v}^{\prime}(T)$ be the level surfaces of $t, u$ and $v$ inside $V_{T}$, we will put the superscripts "i" and "e" on them to denote their intersections with $V_{T}^{i}$ and $V_{T}^{e}$, e.g. $C_{u}^{e}(T)=C_{u} \cap V_{T}^{e}$. We will omit the index $T$ if $T=\infty$, for example, $V=V_{\infty}$, $C_{u}^{e}=C_{u}^{e}(\infty)$ etc.

For any spin- $n / 2$ field $\psi_{A B \ldots}$, define the following energy norms:

$$
\begin{aligned}
& Q_{0}(t)=Q_{0}(\psi)(t)=\int_{\Sigma_{t}} Q_{\mu \nu \ldots \alpha}(\psi) T^{a} \bar{K}_{0}^{\mu} \cdots \bar{K}_{0}^{v}, \\
& \tilde{Q}_{0}(u)=\tilde{Q}_{0}(\psi)(u)=\int_{C_{u}} Q_{\mu v \ldots \alpha}(\psi) l^{\alpha} \bar{K}_{0}^{\mu} \cdots \bar{K}_{0}^{v}, \\
& \tilde{Q}_{0}^{\prime}(u)=\tilde{Q}_{0}^{\prime}(\psi)(v)=\int_{C_{v}^{\prime}} Q_{\mu \nu \ldots \alpha}(\psi) l^{\prime \alpha} \bar{K}_{0}^{\mu} \cdots \bar{K}_{0}^{v} .
\end{aligned}
$$

The integrals on the null hypersurfaces $C_{u}$ and $C_{v}^{\prime}$ are defined as follows:

For any given null hypersurface $\mathscr{N}$ in a space time, let $l$ be a generator of $\mathscr{N}$; it is determined up to the rescaling

$$
l \rightarrow a l,
$$

where $a$ is a nonzero real function on $\mathscr{N}$. If $f$ is a scalar function on $\mathscr{N}$, which depends on the choice of the generator $l$ and which under the rescaling (47), $f \rightarrow a f$. Then define the integral to be

$$
\int_{\mathscr{N}} f d V_{. N}=\int_{a}^{b} d r \int_{S_{r}} f d S_{r}
$$

where $r$ is the affine parameter of $\mathscr{N}$, that is $l(r)=1 ; S_{r}$ is the level surface on $\mathscr{N}$ of constant $r ; S_{\alpha}$ and $S_{b}$ are the boundary of $\mathscr{N}$. Clearly the integrands in the definition of $\tilde{Q}_{0}(u)$ and $\tilde{Q}_{0}^{\prime}(v)$ have the right scaling, thus the integrals are well defined.

Similarly we also define the norms on other regions such as on $\Sigma_{t}^{i}, C_{u}^{e}(T)$ and $C_{v}^{\prime e}(T)$. Since $T, l, l^{\prime}, \bar{K}_{0}$ are all future directed, from Lemma 1 , these energy norms are all positive. Later in Theorem 2 we will prove they actually bound from below by some weighted Sobolev norms.

For $s=0,1, \ldots$, let

$$
Q_{s}^{*}(T)=\sup _{0<t<T} Q_{s}(t)+\sup _{-\infty<u<\infty} \tilde{Q}_{s}(u, T)+\sup _{0<v<\infty} \tilde{Q}_{s}^{\prime}(v, T),
$$

where $Q_{s}(t)$ are defined inductivity as follows,

$$
Q_{s+1}(\psi)(t)=Q_{s}(\psi)(t)+Q_{s}\left(\hat{\mathscr{L}}_{T} \psi\right)(t)+Q_{s}\left(\hat{\mathscr{L}}_{\mathcal{O}} \psi\right)(t)+Q_{s}\left(\hat{\mathscr{L}}_{S} \psi\right)(t),
$$

where $Q_{s}\left(\hat{\mathscr{L}}_{\mathscr{O}} \psi\right)(t)=\sum_{i, j=1}^{3} Q_{s}\left(\hat{\mathscr{L}}_{\Omega_{i j}} \psi\right)(t)$. Similarly define $\tilde{Q}_{s}(u, T)$ and $\tilde{Q}_{s}^{\prime}(v, T)$ by using the null hypersurfaces $C_{u}(T)$ and $C_{v}^{\prime}(T)$ respectively. 
Let

$$
R_{s}^{*}(T)=\sum_{k=0}^{s}\left\{\sup _{0<t<T} R_{k}^{i}(t)+\sup _{-\infty<u<\infty} \tilde{R}_{k}^{e}(u, T)+\sup _{0<v<\infty} \tilde{R}_{k}^{\prime e}(v, T)\right\},
$$

where each term is defined as follows:

$$
\begin{aligned}
R_{s}^{i}(t) & =\int_{\Sigma_{t}^{i}}(1+t)^{2(n+s-1)}\left|\nabla^{s} \psi\right|^{2}, \\
\tilde{R}_{s}^{e}(u, T) & =\int_{C_{u}^{e}(T)} \sum_{k=-n / 2+1} \sum_{l+m=s} \tau_{+}^{n+2(k+s-1)} \tau_{-}^{n-2 k}\left|D^{l} \nabla^{m} \psi_{k}\right|^{2}, \\
\tilde{R}_{s}^{\prime e}(v, T) & =\int_{C_{v}^{\prime e}(T)} \sum_{k=-n / 2}^{n / 2-1} \sum_{l+m=s} \tau_{+}^{n+2(k+m)} \tau_{-}^{n+2(l-k-1)}\left|D^{\prime l} \nabla^{m} \psi_{k}\right|^{2},
\end{aligned}
$$

where

$$
\begin{aligned}
\left|\nabla^{m} \psi\right|^{2} & =\sum_{i+j=m}\left|\partial^{i} \not^{\prime j} \psi\right|^{2} \\
\left|\nabla^{s} \psi\right|^{2} & =\left|\nabla^{s} \psi_{A B \ldots}\right|^{2}=\sum_{i=1}^{3} \sum_{\alpha_{1}+\alpha_{2}+\alpha_{3}=s}\left|\nabla_{i}^{\alpha_{i}} \psi_{A B \ldots}\right|^{2} \\
\left|\psi_{A B \ldots}\right|^{2} & =\sigma_{0}^{A A^{\prime}} \sigma_{0}^{B B^{\prime}} \ldots \psi_{A B \ldots} \bar{\psi}_{A^{\prime} B^{\prime} \ldots} .
\end{aligned}
$$

Actually we can also bound from below the following norm on the exterior region of each time slice, (but we are not going to use this fact),

$$
\begin{aligned}
\tilde{R}_{s}^{e}(t)= & \int_{\Sigma_{t}^{e}} \sum_{k=-n / 2+1}^{n / 2} \sum_{l+m=s} \tau_{+}^{n+2(k+s-1)} \tau_{-}^{n-2 k}\left|D^{l} \nabla^{m} \psi_{k}\right|^{2} \\
& +\sum_{k=-n / 2}^{n / 2-1} \sum_{l+m=s} \tau_{+}^{n+2(k+m)} \tau_{-}^{n+2(l-k-1)}\left|D^{\prime l} \nabla^{m} \psi_{k}\right|^{2} .
\end{aligned}
$$

Theorem 2. If $\psi$ satisfies the spin-n/2 Eq. (10) in $V_{T}$, then

$$
R_{s}^{*}(T) \leqq C Q_{s}^{*}(T) \leqq C Q_{s}(0),
$$

where $C$ is a constant independent of $T$ and the initial data.

Proof. $Q_{s}^{*}(T) \leqq C Q_{s}(0)$ is direct result of Lemma 1 and Lemma 5. To prove $R_{s}^{*}(T) \leqq C Q_{s}^{*}(T)$, we do induction with respect to $s=0,1,2, \ldots$. Since the norms $\tilde{R}_{s}^{e}(u, T)$ and $\tilde{R}_{s}^{\prime e}(v, T)$ are symmetric, we only need to estimate one of them, for instance, take $\widetilde{R}_{s}^{e}(u, T)$.

When $s=0$, use

$$
\bar{K}_{0}=\frac{1}{\sqrt{2}}\left(\tau_{+}^{2} l+\tau_{-}^{2} l^{\prime}\right)
$$

to compute

$$
Q\left(l, \bar{K}_{0}, \ldots, \bar{K}_{0}\right)=2^{(1-n) / 2} \sum_{k=0}^{n-1}\left(\begin{array}{c}
n-1 \\
k
\end{array}\right) \tau_{+}^{2 k} \tau_{-}^{2(n-k-1)} Q(\underbrace{l, \ldots, l}_{k+1 \text { times }}, \underbrace{l^{\prime}, \ldots, l^{\prime}}_{n-k-1 \text { times }})
$$




$$
\begin{aligned}
& =2^{(1-n) / 2} \sum_{k=0}^{n-1}\left(\begin{array}{c}
n-1 \\
k
\end{array}\right) \tau_{+}^{2 k} \tau_{-}^{2(n-k-1)}\left|\psi_{k+1-n / 2}\right|^{2} \\
& \geqq C \sum_{k=-n / 2+1}^{n / 2} \tau_{+}^{n+2(k-1)} \tau_{-}^{n-2 k}\left|\psi_{k}\right|^{2} .
\end{aligned}
$$

Therefore we have

$$
\tilde{R}_{0}^{e}(u, T) \leqq C \widetilde{Q}_{0}^{e}(u, T)
$$

For $R_{0}^{i}(t)$, since in $V^{i}, \tau_{-} \geqq C(1+t)$, and use $T=\frac{1}{\sqrt{2}}\left(l+l^{\prime}\right)$,

$$
\begin{aligned}
Q\left(T, \bar{K}_{0}, \ldots, \bar{K}_{0}\right) & =\frac{1}{\sqrt{2}}\left[Q\left(l, \bar{K}_{0}, \ldots, \bar{K}_{0}\right)+Q\left(l^{\prime}, \bar{K}_{0}, \ldots, \bar{K}_{0}\right)\right] \\
& \geqq C(1+t)^{2(n-1)} \sum_{k=-n / 2}^{n / 2}\left|\psi_{k}\right|^{2} \\
& \geqq C(1+t)^{2(n-1)}\left|\psi_{A B \ldots}\right|^{2} .
\end{aligned}
$$

Thus

$$
R_{0}^{i}(t) \leqq C Q_{0}^{i}(t)
$$

Suppose that the theorem is true for some $s \geqq 0$, we want to prove that it is also true for $s+1$.

Let $\phi_{A B \ldots}=\hat{\mathscr{L}}_{T}^{i} \hat{\mathscr{L}}_{S}^{j} \hat{\mathscr{L}}_{\mathcal{O}}^{l} \psi_{A B \ldots}$, where $i+j+l=s$, then since

$$
\left|\nabla \phi_{k}\right|^{2} \leqq \frac{C}{r^{2}}\left(\left|\hat{\mathscr{P}}_{\mathcal{O}} \phi_{k}\right|^{2}+\left|\phi_{k}\right|^{2}\right)
$$

by induction, we know

$$
\int_{C_{u}^{e}(T)} \sum_{k=-n / 2+1}^{n / 2} \sum_{l+m=s} \tau_{+}^{n+2(k+s)} \tau_{-}^{n-2 k}\left|D^{l} \nabla^{m+1} \psi_{k}\right|^{2} \leqq C Q_{s+1}^{*}(T) .
$$

Use (33) to obtain the following estimate,

$$
\int_{C_{u}^{e}(T)} \sum_{k=-n / 2+1}^{n / 2-1} \sum_{l+m=s} \tau_{+}^{n+2(k+s+1)} \tau_{-}^{n-2(k+1)}\left|D^{l+1} \nabla^{m} \psi_{k}\right|^{2} \leqq C Q_{s+1}^{*}(T) .
$$

The estimate for $D^{l+1} \nabla^{m} \psi_{n / 2}$ is still missing, but (33) gives us

$$
\int_{C_{u}^{e}(T)} \tau_{+}^{2(n+s-1)} \tau_{-}^{2}\left|D^{l} \nabla^{m} D^{\prime} \psi_{n / 2}\right|^{2} \leqq C Q_{s+1}^{*}(T)
$$

by induction we also have the estimate for

$$
\begin{aligned}
& \hat{\mathscr{A}}_{T} \phi_{n / 2}=\frac{1}{\sqrt{2}}\left(D \phi_{n / 2}+D^{\prime} \phi_{n / 2}\right), \\
& \hat{\mathscr{A}}_{S} \phi_{n / 2}=\frac{1}{\sqrt{2}}\left(v D \phi_{n / 2}+u D^{\prime} \phi_{n / 2}\right)+\frac{n+2}{8} \phi_{n / 2} .
\end{aligned}
$$


Therefore we have

Thus

$$
\int_{C_{u}^{e}(T)} \sum_{l+m=s} \tau_{+}^{2(n+s)}\left|D^{l+1} \nabla^{m} \psi_{n / 2}\right|^{2} \leqq C Q_{s+1}^{*}(T) .
$$

$$
\tilde{R}_{s+1}^{e}(u, T) \leqq C \tilde{Q}_{s+1}^{e}(u, T) .
$$

Because $\Omega_{i j}$ is degenerate on the spatial central line, to estimate the norm in the interior, we will use the following lemma:

Lemma 6. Let $\psi_{A B \ldots}$ be a spin field on a time slice $\Sigma=\Sigma_{t}$, assume it has compact support, let $\mathscr{D}$ be the Dirac operator on $\Sigma$, that is, $\mathscr{D} \psi=\sigma^{i A A^{\prime}} D_{i} \psi_{A B \ldots}$, then

$$
\int_{\Sigma}|\nabla \psi|^{2} \leqq 2 \int_{\Sigma}|\mathscr{D} \psi|^{2}
$$

Proof.

$$
\begin{aligned}
& \int_{\Sigma}|\mathscr{D} \psi|^{2}=\int_{\Sigma} \sigma^{i A C^{\prime}} D_{i} \bar{\psi}_{C^{\prime} B^{\prime} \ldots} \sigma^{j C A^{\prime}} D_{j} \psi_{C B \ldots} \sigma_{0 A A^{\prime}} \sigma_{0}^{B B^{\prime}} \ldots \\
& =-\int_{\Sigma} \sigma^{i A C^{\prime}} \sigma^{j C A^{\prime}} \sigma_{0 A A^{\prime}} \bar{\psi}_{C^{\prime} B^{\prime} \ldots} D_{i} D_{j} \psi_{C B \ldots} \sigma_{0}^{B B^{\prime}} \ldots \\
& =\frac{1}{2} \int_{\Sigma} \sigma^{i A C^{\prime}} \sigma_{A A^{\prime}}^{j} \sigma_{0}^{C A^{\prime}} \bar{\psi}_{C^{\prime} B^{\prime} \ldots}\left(D_{i} D_{j}+D_{j} D_{i}+\left[D_{i}, D_{j}\right]\right) \psi_{C B \ldots} \sigma_{0}^{B B^{\prime}} \ldots \\
& =\frac{1}{2} \int_{\Sigma} D_{i} \bar{\psi}_{C^{\prime} B^{\prime} \ldots} D_{j} \psi_{C B \ldots}\left(\sigma^{i A C^{\prime}} \sigma_{A A^{\prime}}^{j}+\sigma^{j A C^{\prime}} \sigma_{A A^{\prime}}^{i}\right) \sigma_{0 A A^{\prime}} \sigma_{0}^{B B^{\prime}} \ldots \\
& \geqq \frac{1}{2} \int_{\Sigma}|\nabla \psi|^{2}
\end{aligned}
$$

Now we continue the proof of Theorem 2. Let $\tilde{\phi}_{A B \ldots}=\eta\left(\frac{r}{1+t}\right) \phi_{A B \ldots}$, where $\eta(s)$ is a cut-off function such that

then

$$
\eta(s)=\left\{\begin{array}{lll}
1 & \text { if } & s<1 / 3 \\
0 & \text { if } & s>1 / 2
\end{array}\right.
$$

$$
\begin{aligned}
\mathscr{D} \tilde{\phi}_{A B \ldots}= & {\left[\sigma^{i A A^{\prime}} \nabla_{i} \eta\left(\frac{r}{1+t}\right)\right] \phi_{A B \ldots} } \\
& -\sigma_{0}^{A A^{\prime}} \frac{\eta\left(\frac{r}{1+t}\right)}{1+t}\left(\hat{\mathscr{L}}_{T}+\hat{\mathscr{L}}_{S}-x^{j} \nabla_{j}-\frac{n+2}{8}\right) \phi_{A B \ldots}
\end{aligned}
$$

Thus

$$
|\mathscr{D} \tilde{\phi}| \leqq \frac{C}{1+t}\left(\left|\hat{\mathscr{L}}_{T} \phi\right|+\left|\hat{\mathscr{L}}_{S} \phi\right|+|\phi|\right)+\frac{1}{3}|\nabla \tilde{\phi}|
$$

The desired estimate in the interior is then an easy consequence of Lemma 6. 
Finally, we want to prove the Sobolev inequalities, which will also be used in the next section, which will give us the proof of Theorem 1 from Theorem 2.

Isoperimetric Inequality.

$$
\int_{S}|f-\bar{f}|^{2} \leqq C\left(\int_{S}|\nabla f|\right)^{2}
$$

where $S$ is any two dimensional sphere; $C$ is independent of the radius of $S ; \bar{f}$ is the average of $f$ over $S$.

\section{Lemma 7.}

$$
\begin{aligned}
& \sup _{-\infty<u<\infty}\left[\left(\int_{C_{u}^{e}(T)} r^{6}|f|^{6}\right)^{1 / 6}+\sup _{\tilde{S}_{u, r} \subset C_{u}^{e}(T)}\left(\int_{\tilde{S}_{u, r}} r^{4}|f|^{4}\right)^{1 / 4}\right] \\
& \leqq C\left(\int_{\Sigma_{0}}|f|^{2}+\left(1+r^{2}\right)|\nabla f|^{2}\right)^{1 / 2}+C \sup _{0<t<T}\left(\int_{\Sigma_{t}^{1}}|f|^{2}+\tau_{-}^{2}|\nabla f|^{2}\right)^{1 / 2} \\
& +C \sup _{-\infty<u<\infty}\left(\int_{C_{u}^{e}(T)}|f|^{2}+r^{2}|D f|^{2}+r^{2}|\nabla f|^{2}\right)^{1 / 2} .
\end{aligned}
$$

We also have a similar inequality on another family of light cones $C_{v}^{\prime}$, its degenerate version is

$$
\begin{aligned}
& \sup _{0<v<\infty}\left[\left(\int_{C_{v}^{\prime e}(T)} r^{4} \tau_{-}^{2}|f|^{6}\right)^{1 / 6}+\sup _{\tilde{S}_{u, r}^{\prime} \subset C_{v}^{\prime e}(T)}\left(\int_{\tilde{S}_{u, r}^{\prime}} r^{2} \tau_{-}^{2}|f|^{4}\right)^{1 / 4}\right] \\
& \leqq C\left(\int_{\Sigma_{0}}|f|^{2}+\left(1+r^{2}\right)|\nabla f|^{2}\right)^{1 / 2} \\
& +C\left(\int_{C_{v}^{\prime e}(T)}|f|^{2}+r^{2}|\nabla f|^{2}+\tau_{-}^{2}\left|D^{\prime} f\right|^{2}\right)^{1 / 2} .
\end{aligned}
$$

Proof. Let $\tilde{S}_{u, r}$ be the radius $r$ sphere on the null hypersurface $C_{u}^{e}(T)$; let $r_{m}(u)$ and $r_{M}(u)$ be respectively the minimum and maximal radii of all such two spheres. Then $\tilde{S}_{u, r_{m}(u)}$ is either in the interior region $V_{T}^{i}$ or on the initial hypersurface $\Sigma_{0} ; \tilde{S}_{u, r_{M}(u)}$ always lies on $\Sigma_{T}$. Apply the Isoperimetric Inequality (52) to $r^{3}|f|^{3}$ on each $\tilde{S}_{u, r}$, use the Hölder inequality, then integrate with respect to $r$ from $r_{m}(u)$ to $r_{M}(u)$, we obtain,

$$
\int_{C_{u}^{e}(T)} r^{6}|f|^{6} \leqq C \sup _{r_{m}(u)<r<r_{M}(u)}\left(\int_{\tilde{S}_{u, r}} r^{4}|f|^{4}\right)\left(\int_{C_{u}^{e}(T)}|f|^{2}+r^{2}|\nabla f|^{2}\right) .
$$

On the other hand,

$$
\begin{aligned}
\int_{\tilde{S_{u}, r}} r^{4}|f|^{4} & \leqq \int_{\tilde{S}_{u, r_{m}(u)}} r^{4}|f|^{4}+C \int_{C_{u}^{e}(T)} r^{4}|f|^{3}|D f|+r^{3}|f|^{4} \\
& \leqq \int_{\tilde{S}_{u, r_{m}(u)}} r^{4}|f|^{4}+C\left(\int_{C_{u}^{e}(T)} r^{6}|f|^{6}\right)^{1 / 2}\left(\int_{C_{u}(T)}|f|^{2}+r^{2}|D f|^{2}\right)^{1 / 2} .
\end{aligned}
$$


Therefore we have

$$
\begin{aligned}
& \left(\int_{C_{u}^{e}(T)} r^{6}|f|^{6}\right)^{1 / 6}+\sup _{r_{m}(u)<r<r_{M}(u)}\left(\int_{\tilde{S}_{u, r}} r^{4}|f|^{4}\right)^{1 / 4} \\
& \leqq\left(\int_{\tilde{S}_{u, r_{m}(T)}} r^{4}|f|^{4}\right)^{1 / 4}+\left(\int_{C_{u}^{e}(T)}|f|^{2}+r^{2}|\nabla f|^{2}+r^{2}|D f|^{2}\right)^{1 / 2} .
\end{aligned}
$$

Therefore we have

$$
\begin{aligned}
& \left(\int_{C_{u}^{e}(T)} r^{6}|f|^{6}\right)^{1 / 6}+\sup _{r_{m}(u)<r<r_{M}(u)}\left(\int_{\tilde{S_{u, r}}} r^{4}|f|^{4}\right)^{1 / 4} \\
& \leqq\left(\int_{\tilde{S}_{u, r_{m}(T)}} r^{4}|f|^{4}\right)^{1 / 4}+\left(\int_{C_{u}^{e}(T)}|f|^{2}+r^{2}|\nabla f|^{2}+r|D f|^{2}\right)^{1 / 2} .
\end{aligned}
$$

But the same proof as above easily gives us the following inequality,

$$
\left(\int_{\tilde{S}_{u, r_{m}(u)}} r^{4}|f|^{4}\right)^{1 / 4} \leqq\left(\int_{\Sigma_{0}}|f|^{2}+\left(1+r^{2}\right)|\nabla f|^{2}+\sup _{0<t<T} \int_{\Sigma_{t}^{t}}|f|^{2}+\left(1+t^{2}\right)|\nabla f|^{2}\right)^{1 / 2} \text {. }
$$

Therefore we have proved (53). Equation (54) is proved in exactly the same way, one just replaces $C_{u}^{e}(T)$ by $C_{v}^{\prime e}(T)$, and replaces $r^{3}|f|^{3}$ by $r^{2} \tau_{-}|f|^{3}$ in (55) before using the Isoperimetric inequality.

Lemma 8. There is a constant $C<\infty$ which is independent of $T$ and $f$ such that,

$$
\begin{aligned}
\sup _{V_{T}} \tau_{+}^{3 / 2}|f| \leqq & C\left(\int_{\Sigma_{0}}|f|^{2}+\left(1+r^{2}\right)|\nabla f|^{2}+\left(1+r^{2}\right)^{2}\left|\nabla^{2} f\right|^{2}\right)^{1 / 2} \\
& +C \sup _{0<t<T}\left(\int_{\Sigma_{t}^{i}}|f|^{2}+\left(1+t^{2}\right)|\nabla f|^{2}+\left(1+t^{2}\right)^{2}\left|\nabla^{2} f\right|^{2}\right)^{1 / 2} \\
& +\sup _{-\infty<u<\infty}\left(\int_{C_{u}^{e}(T)}|f|^{2}+r^{2}|\nabla f|^{2}+r^{2}|D f|^{2}\right. \\
& \left.+r^{4}\left|\nabla^{2} f\right|^{2}+r^{4}|D \nabla f|^{2}\right)^{1 / 2}, \\
\sup _{V_{T} \tau_{+} \tau_{-}^{1 / 2}|f| \leqq} & C \sup _{0<t<T}\left(\int_{\Sigma_{0}}|f|^{2}+\left(1+r^{2}\right)|\nabla f|^{2}+\left(1+r^{2}\right)^{2}\left|\nabla^{2} f\right|^{2}\right)^{1 / 2} \\
& +\sup _{0<v<\infty}\left(\int_{C_{v}^{\prime}(T)}|f|^{2}+r^{2}|\nabla f|^{2}+\tau_{-}^{2}\left|D^{\prime} f\right|^{2}\right. \\
& \left.+r^{4}\left|\nabla^{2} f\right|^{2}+r^{2} \tau_{-}^{2}\left|D^{\prime} \nabla f\right|^{2}\right)^{1 / 2} .
\end{aligned}
$$

This lemma is evident from the following Sobolev inequality: 
Sobolev Inequality.

$$
\sup _{S}|f| \leqq C r^{-1 / 2}\left(\int_{S}|f|^{4}+r^{4}|\nabla f|^{4}\right)^{1 / 4}
$$

where $C$ is independent of the radius of $S$.

Proof of Theorem 1. From Lemma 8, we have the following estimate:

$$
[\psi]^{2}(T) \leqq C R_{2}^{*}(T) .
$$

Therefore Theorem 1 follows from Theorem 2.

\section{Yang-Mills Equations}

It is easier to state the initial data for the Cauchy problem in the language of tensors because the spinor structure are naturally adapted to the null structure of the space time. It will be more convenient to use spinors if we want to solve the Goursat problem.

Let $\left(\varphi_{i}(x), E_{i}(x)\right)$ be the initial data, $x \in \mathbf{R}^{3}, \varphi_{i}(x)$ is the gauge potential of a gauge field on $\mathbf{R}^{3}, E_{i}$ is a gauge covariant one form. It has to satisfy the following constraint equation:

$$
\underline{\nabla}^{i} E_{i}=\nabla^{i} E_{i}+\left[\varphi^{i}, E_{i}\right]=0,
$$

where $\underline{\nabla}$ means the gauge covariant derivative on $\mathbf{R}^{3}$ with respect to $\varphi_{i}(x)$.

The curvature tensor $F_{\mu \nu}$, and thus its equivalent spinor form $\Psi_{A B}$, on the initial surface $\Sigma_{0}$ can easily be expressed by the initial data as follows:

$$
F_{i 0}(0, x)=E_{i}(x), \quad F_{i j}(0, x)=\partial_{i} \varphi_{j}(x)-\partial_{j} \varphi_{i}(x)+\left[\varphi_{i}, \varphi_{j}\right] .
$$

Except for the initial data, everything else in this section will always be gauge covariant, i.e. we will never refer to any quantity which is gauge dependent. For gauge covariant quantities, we will adopt as before the same notations and concepts such as Lie derivative, energy norms, etc., just simply replacing the usual derivative by the gauge covariant derivative.

The goal is to prove the following theorem:

Theorem 3. Suppose the initial data are smooth, satisfy the constraint equation and the following smallness condition:

$$
\|\Psi(0, x)\|_{H_{2,1}} \leqq \varepsilon_{0},
$$

where $\varepsilon_{0}$ is a small constant, $H_{2,1}$ is the gauge covariant weighted Sobolev norm (see (3) in Sect. 1). Then there exists a unique (up to gauge transformation) global solution of Yang-Mills equations in Minkowski space; moreover it has the following decay estimate,

$$
[\Psi] \leqq C\|\Psi(0, x)\|_{H_{2,1}} .
$$

Almost the entire section is devoted to the proof of this theorem; the case when the initial data has charge will be discussed in the end. The proof of local existence is rather standard (see [5] or [7]). The following conclusion is also simple to prove $([6])$ : 
The solution blows up at finite time $T_{0}<\infty$ if and only if $\sup _{V_{T_{0}}}|\Psi|=\infty$.

So from now on we are always assumed to be given a guage field in $V_{T}$ for some $T>0$. Let $\Psi_{A B}$ be its curvature spinor, it satisfies the gauge covariant spin-1 equations.

Lemma 9. For any gauge invariant spin-1 field $\psi_{A B}$ in $V_{T}$, then

$$
\begin{aligned}
\mathbf{D}^{A A^{\prime}} \hat{\mathscr{L}}_{X} \psi_{A B}= & \hat{\mathscr{L}}_{X} \mathbf{D}^{A A^{\prime}} \psi_{A B}+X_{C}^{A^{\prime}}\left[\Psi^{A C}, \psi_{A B}\right]+X_{C^{\prime}}^{A}\left[\bar{\Psi}^{A^{\prime} C^{\prime}}, \psi_{A B}\right] \\
& +\frac{1}{2} \hat{\pi}^{A C A^{\prime} C^{\prime}} D_{C C^{\prime}} \psi_{A B}+\frac{1}{4}\left(D_{C C^{\prime}} \hat{\pi}^{A C A^{\prime} C^{\prime}} \psi_{A B}\right) \\
& -\frac{1}{4}\left(\psi_{A C} D_{B}^{C^{\prime}} \hat{\pi}_{C^{\prime}}^{A C A^{\prime}}+\psi_{A C} D^{C C^{\prime}} \hat{\pi}_{B C^{\prime}}^{A A^{\prime}}\right) .
\end{aligned}
$$

The right-hand side of (64) will be denoted by $J_{B}^{A^{\prime}}(X, \psi)$. We will also denote $J_{\mu}(X)=J_{\mu}(X, \Psi)$, and $J_{\mu}(X, Y)=J_{\mu}\left(X, \hat{\mathscr{L}}_{Y} \Psi\right)$.

Proof.

$$
\begin{aligned}
\mathbf{D}^{A A^{\prime}} \mathscr{L}_{X} \psi_{A B}= & \mathbf{D}^{A A^{\prime}}\left\{X^{\mu} \mathbf{D}_{\mu} \psi_{A B}+h_{A}^{C} \psi_{C B}+h_{B}^{C} \psi_{A C}\right\} \\
= & X^{\mu} \mathbf{D}_{\mu} \mathbf{D}^{A A^{\prime}} \psi_{A B}+X_{C C^{\prime}}\left[\mathbf{D}^{A A^{\prime}}, \mathbf{D}^{C C^{\prime}}\right] \psi_{A B} \\
& +\frac{1}{2} \pi^{A A^{\prime} C C^{\prime}} \mathbf{D}_{C C^{\prime}} \psi_{A B}+\left(h^{A C} \varepsilon^{A^{\prime} C^{\prime}}+\bar{h}^{A^{\prime} C^{\prime}} \varepsilon^{A C}\right) \mathbf{D}_{C C^{\prime}} \psi_{A B} \\
& +\mathbf{D}^{A A^{\prime}}\left\{h_{A}^{C} \psi_{C B}+h_{B}^{C} \psi_{A C}\right\} \\
= & X^{\mu} \mathbf{D}_{\mu} \mathbf{D}^{A A^{\prime}} \psi_{A B}+X_{C}^{A^{\prime}}\left[\Psi^{A C}, \psi_{A B}\right]+X_{C^{\prime}}^{A}\left[\bar{\Psi}^{A^{\prime} C^{\prime}}, \psi_{A B}\right] \\
& +\mathbf{D}^{A A^{\prime}}\left\{h_{A}^{C} \psi_{C B}+h_{B}^{C} \psi_{A C}\right\} .
\end{aligned}
$$

The rest of the proof is the same as Lemma 5.

Lemma 10. For any gauge invariant spin-1 field $\psi_{A B}$, if $\mathbf{D}_{A^{\prime}}^{A} \psi_{A B}=J_{B A^{\prime}}, J_{\mu}$ is some gauge covariant one form, then 1.

$$
\begin{aligned}
Q_{0}^{*}(\psi)(T) \leqq & C Q_{0}(\psi)(0)+C \int_{V_{T}^{\mathrm{i}}}\left(1+t^{2}\right)|\psi||J| \\
& +C \int_{V_{T}^{e}} \tau_{+}^{2}\left(\left|\psi_{1}\right|\left|J_{-1}^{\prime}\right|+\left|\psi_{0}\right|\left|J_{0}\right|\right) \\
& +C \int_{V_{T}^{e}} \tau_{-}^{2}\left(\left|\psi_{-1}\right|\left|J_{-1}\right|+\left|\psi_{0}\right|\left|J_{0}^{\prime}\right|\right)
\end{aligned}
$$

where $\psi_{k}$ and $J_{k}$ were defined in (21),(22).

2.

$$
\begin{aligned}
\left|J_{0}(\mathcal{O}, \psi)\right| \leqq C r & \left(\left|\Psi_{1}\right|\left|\psi_{0}\right|+\left|\Psi_{0}\right|\left|\psi_{1}\right|\right)+\left|\hat{\mathscr{P}}_{\mathcal{O}} J_{0}\right|, \\
\left|J_{-1}^{\prime}(\mathcal{O}, \psi)\right| \leqq C r & \left(\left|\Psi_{-1}\right|\left|\psi_{1}\right|+\left|\Psi_{0}\right|\left|\psi_{0}\right|\right)+\left|\hat{\mathscr{P}}_{\mathcal{O}} J_{-1}^{\prime}\right|, \\
\left|J_{0}(T, \psi)\right|+\left|J_{0}(S, \psi)\right| \leqq & C \tau_{+}\left|\Psi_{1}\right|\left|\psi_{1}\right|+C \tau_{-}\left(\left|\Psi_{-1}\right|\left|\psi_{1}\right|+\left|\Psi_{0}\right|\left|\psi_{0}\right|\right) \\
& +\left|\hat{\mathscr{P}}_{T} J_{0}\right|+\left|\hat{\mathscr{P}}_{S} J_{0}\right|, \\
\left|J_{-1}^{\prime}(T, \psi)\right|+\left|J_{-1}^{\prime}(S, \psi)\right| \leqq & C \tau_{+}\left(\left|\Psi_{0}\right|\left|\psi_{1}\right|+\left|\Psi_{1}\right|\left|\psi_{0}\right|\right)+C \tau_{-}\left|\Psi_{-1}\right|\left|\psi_{0}\right| \\
& +\left|\hat{\mathscr{P}}_{T} J_{-1}^{\prime}\right|+\left|\hat{\mathscr{P}}_{S} J_{-1}^{\prime}\right| .
\end{aligned}
$$

By taking the prime, we get the corresponding estimates of $J_{0}^{\prime}$ and $J_{-1}$. In particular, 
in $V_{T}^{i}$, for $X=\Omega_{i j}, T, S$,

$$
|J(X, \psi)| \leqq C(1+t)|\Psi||\psi|+C(1+t)|\mathbf{D} J| .
$$

Proof. This lemma can be proved either by the direct calculation or by using the weights. In the following we will prove 1 by direct calculation and 2 by using the weights:

$$
\begin{aligned}
D^{\mu} Q_{\mu \nu}(\psi) & =\left(\mathbf{D}^{A A^{\prime}} \psi_{A B}, \bar{\psi}_{A^{\prime} B^{\prime}}\right)+\left(\psi_{A B}, \mathbf{D}^{A A^{\prime}} \bar{\psi}_{A^{\prime} B^{\prime}}\right) \\
& =\left(J_{B}^{A^{\prime}}, \bar{\psi}_{A^{\prime} B^{\prime}}\right)+\left(\psi_{A B}, \bar{J}_{B^{\prime}}^{A}\right) .
\end{aligned}
$$

Therefore

$$
\begin{aligned}
Q_{0}^{*}(\psi)(T) \leqq & C Q_{0}(\psi)(0)+C \int_{V_{T}} \mid \bar{K}_{0}^{B B^{\prime}}\left[\left(\bar{\psi}_{A^{\prime} B^{\prime}}, J_{B}^{A^{\prime}}\right)+\left(\psi_{A B}, \bar{J}_{B^{\prime}}^{A}\right)\right] \\
\leqq & C Q_{0}(\psi)(0)+C \int_{V_{T}^{i}}\left(1+t^{2}\right)|\psi||J| \\
& +C \int_{V_{T}^{e}} \tau_{+}^{2}\left(\left|\xi^{B} \xi^{A} \psi_{A B}\right|\left|\bar{\xi}^{B^{\prime}} \eta^{A} \bar{J}_{A B^{\prime}}\right|+\left|\xi^{B} \eta^{A} \psi_{A B}\right|\left|\bar{\xi}^{B^{\prime}} \xi^{A} \bar{J}_{A B^{\prime}}\right|\right) \\
& +C \int_{V_{T}^{e}} \tau_{-}^{2}\left(\left|\eta^{B} \xi^{A} \psi_{A B}\right|\left|\bar{\eta}^{B^{\prime}} \eta^{A} \bar{J}_{A B^{\prime}}\right|+\left|\eta^{B} \eta^{A} \psi_{A B}\right|\left|\bar{\eta}^{B^{\prime}} \xi^{A} \bar{J}_{A B^{\prime}}\right|\right) \\
\leqq & C Q_{0}(0)+C \int_{V_{T}^{i}}\left(1+t^{2}\right)|\psi||J| \\
& +C \int_{V_{T}^{e}} \tau_{+}^{2}\left(\left|\psi_{1}\right|\left|\bar{J}_{-1}^{\prime}\right|+\left|\psi_{0}\right|\left|\bar{J}_{0}\right|\right) \\
& +C \int_{V_{T}^{e}} \tau_{-}^{2}\left(\left|\psi_{0}\right|\left|\bar{J}_{0}^{\prime}\right|+\left|\psi_{-1}\right|\left|\bar{J}_{-1}\right|\right) .
\end{aligned}
$$

In order to prove 2, remark that from Lemma 9 we know that for the conformal Killing vector fields $X=\Omega_{i j}, T$ or $S, J_{k}(X, \psi)-\mathscr{L}_{X} J_{k}$ is the linear combination of $\left[\Psi_{i}, \psi_{j}\right]$ and $\left[\bar{\Psi}_{i}, \psi_{j}\right]$ with coefficients $X^{m}$.

Let $X=\Omega_{i j}=a_{1} m+a_{2} m^{\prime}$, the weights of $a_{1}$ and $a_{2}$ are $(-1,1)$ and $(1,-1)$ respectively, and $\left|a_{1}\right|+\left|a_{2}\right| \leqq C r$. Because the weight of $J_{0}\left(\Omega_{i j}, \psi\right)-\mathscr{A}_{\Omega_{i}} J_{0}$ is $(1,1)$, the only terms of form $\left[\Psi_{i}, \psi_{j}\right]$ and $\left[\bar{\Psi}_{i}, \psi_{j}\right]$, which can possibly appear with the coefficient $a_{1}$ have to have weight $(2,0)$, so they can only be $\left[\Psi_{1}, \psi_{0}\right]$ and $\left[\Psi_{0}, \psi_{1}\right]$; in the same way, the only term that can appear with the coefficient $a_{2}$ has weight $(0,2)$, so it must only be $\left[\bar{\Psi}_{1}, \psi_{0}\right]$. Therefore

$$
\left|J_{0}(\mathcal{O}, \psi)-\hat{\mathscr{L}}_{\mathcal{O}} J_{0}\right| \leqq C r\left(\left|\Psi_{1}\right|\left|\psi_{0}\right|+\left|\Psi_{0}\right|\left|\psi_{1}\right|\right)
$$

For $J_{-1}^{\prime}\left(\Omega_{i j}, \psi\right)$, its weight is $(1,-1)$, thus the only term with coefficient $a_{1}$ is $\left[\bar{\Psi}_{-1}, \psi_{-1}\right]$; the only terms with coefficient $a_{2}$ are $\left[\Psi_{0}, \psi_{0}\right]$ and $\left[\bar{\Psi}_{0}, \psi_{0}\right]$. Therefore

$$
\left|J_{-1}^{\prime}(\mathcal{O}, \psi)-\hat{\mathscr{P}}_{\mathcal{O}} J_{-1}^{\prime}\right| \leqq C r\left(\left|\Psi_{-1}\right|\left|\psi_{1}\right|+\left|\Psi_{0}\right|\left|\psi_{0}\right|\right) .
$$

For $X=T$ or $S, X=a_{1} l+a_{2} l^{\prime}$, the weights of coefficients $a_{1}$ and $a_{2}$ are respectively $(-1,1)$ and $(1,1) ;\left|a_{1}\right| \leqq C \tau_{+},\left|a_{2}\right| \leqq C \tau_{-}$. The rest of the proof is the same as before.

Proof of Theorem 3. From the local existence theorem, we can assume there is a 
solution in $V_{T}$, for some $\infty \geqq T>0$, such that either $T=\infty$ or $\sup |\Psi|=\infty$. Let

$$
T_{0}=\sup \left\{t<T \mid R_{2}^{*}(\Psi)(t)<\varepsilon_{1}^{2}\right\},
$$

where $\varepsilon_{1}$ is a small constant to be fixed later, then

$$
R_{2}^{*}\left(T_{0}\right) \leqq \varepsilon_{1}^{2}
$$

Claim. Under the à priori assumption (73), if $\varepsilon_{1}$ is sufficiently small (independent of $T_{0}$ and the initial data), then there is a constant $C=C\left(\varepsilon_{1}\right)$ such that,

$$
R_{2}^{*}\left(T_{0}\right) \leqq C Q_{2}(0) \text {. }
$$

Choose $\varepsilon_{0}$ such that $2 C \varepsilon_{0}^{2} \leqq \varepsilon_{1}^{2}$. If $Q_{2}(0) \leqq \varepsilon_{0}^{2}$, then $R_{2}^{*}\left(T_{0}\right) \leqq \varepsilon_{2}^{2}<\varepsilon_{1}^{2}$. Therefore $T_{0}=T=\infty$ this proves Theorem 3 .

It remains to prove this claim. The proof is divided into three steps in the following: the first step is to prove the equivalence between the energy norms $Q_{2}^{*}(T)$ and the Sobolev norms $R_{2}^{*}(T)$; the second step is to estimate $Q_{1}^{*}(T)$, the third step is to estimate $Q_{2}^{*}(T)$. Step 1 is rather easy to prove, step 3 is very similar to step 2. All of the estimates are under the à priori assumption of (73).

Step 1. Equivalence of Norms: there is a constant $C$ depending on $\varepsilon_{1}$, such that

$$
R_{2}^{*}(T) \leqq C Q_{2}^{*}(T) \text {. }
$$

As in the proof of Theorem 2, we divide it into an interior and exterior part. The interior part relies on the following lemma which is the gauge covariant version of Lemma 6,

Lemma 11. For a compact supported gauge covariant spin field $\psi_{A B}$ on a time slice $\Sigma$, let $\underline{\nabla}$ denote the gauge covariant derivative on $\Sigma, \mathscr{D}$ the corresponding Dirac operator, then

$$
\int_{\Sigma}|\underline{\nabla} \psi|^{2} \leqq 2 \int_{\Sigma}|\underline{D} \psi|^{2}+C \int_{\Sigma}|\Psi||\psi|^{2}
$$

where $\Psi$ is the curvature spinor of the gauge field.

For the exterior part, the estimate $\tilde{R}_{1}^{e}(u, T)$ and $\tilde{R}_{1}^{\prime e}(v, T)$ is the same as that of Theorem 2 because we only used the Yang-Mills equations without taking any derivative. To estimate $\tilde{R}_{2}^{e}(u, T)$ (same for $\widetilde{R}_{2}^{\prime e}(v, T)$ ), we use (33), (33)' to obtain the following estimate for $X=\Omega_{i j}, T_{0}, S$,

$$
\begin{gathered}
\int_{C_{u}^{e}(T)} \tau_{+}^{2} \tau_{-}^{2}\left|D \hat{\mathscr{P}}_{X} \Psi_{0}\right|^{2} \leqq C Q_{2}^{*}(T)+C \int_{C_{u}^{e}(T)} \tau_{+}^{2} \tau_{-}^{2}\left|J_{0}(X)\right|^{2}, \\
\int_{C_{u}^{e}(T)} \tau_{+}^{2} \tau_{-}^{2}\left|D^{\prime} \hat{\mathscr{P}}_{X} \Psi_{1}\right|^{2} \leqq C Q_{2}^{*}(T)+C \int_{C_{u}^{e}(T)} \tau_{+}^{2} \tau_{-}^{2}\left|J_{-1}^{\prime}(X)\right|^{2} .
\end{gathered}
$$

So one has to estimate the following two integrals for $X=\Omega_{i j}, T_{0}, S$,

$$
\int_{C_{u}^{e}(T)} \tau_{+}^{2} \tau_{-}^{2}\left|J_{0}(X)\right|^{2} \text { and } \int_{C_{u}^{e}(T)} \tau_{+}^{2} \tau_{-}^{2}\left|J_{-1}^{\prime}(X)\right|^{2} .
$$


We use Lemma 10,

$$
\begin{aligned}
& \int_{C_{u}^{e}(T)} \tau_{+}^{2} \tau_{-}^{2} \sum_{X=\Omega_{1 j}, T, S}\left(\left|J_{0}(X)\right|^{2}+\left|J_{-1}^{\prime}(X)\right|^{2}\right) \\
& \quad \leqq C \int_{C_{u}^{e}(T)} \tau_{+}^{4} \tau_{-}^{2}\left(\left|\Psi_{1}\right|^{2}\left|\Psi_{-1}\right|^{2}+\left|\Psi_{1}\right|^{2}\left|\Psi_{0}\right|^{2}+\left|\Psi_{0}\right|^{4}+\left|\Psi_{1}\right|^{4}\right) \\
& \quad+\tau_{+}^{2} \tau_{-}^{4}\left(\left|\Psi_{0}\right|^{2}\left|\Psi_{-1}\right|^{2}+\left|\Psi_{1}\right|^{2}\left|\Psi_{-1}\right|^{2}+\left|\Psi_{0}\right|^{4}\right) \\
& \leqq C \sup _{C_{u}^{e}(T)}\left(r^{2} \tau_{-}^{2}\left|\Psi_{-1}\right|^{2}+r^{4}\left|\Psi_{0}\right|^{2}+r^{4}\left|\Psi_{1}\right|^{2}\right) \int_{C_{u}^{e}(T)} \tau_{+}^{2}\left|\Psi_{1}\right|^{2}+\tau_{-}^{2}\left|\Psi_{0}\right|^{2} \\
& \leqq C[\Psi]^{2} Q_{0}^{*}(T) .
\end{aligned}
$$

Thus

$$
\tilde{R}_{2}^{e}(u, T) \leqq C Q_{2}^{*}(T)+C \varepsilon_{1}^{2} Q_{0}^{*}(T) .
$$

This then completed the proof of Step 1 .

Step 2. Estimate of $Q_{1}^{*}(T)$ : if $\varepsilon_{1}$ is sufficiently small, then

$$
Q_{1}^{*}(T) \leqq C Q_{1}(0) \text {. }
$$

Apply 1 in Lemma 10 for $\psi_{A B}=\hat{\mathscr{L}}_{X} \Psi_{A B}$, where $X=\Omega_{i j}, T, S$, we have to estimate the following error terms:

$$
\begin{aligned}
\mathscr{E}_{1}(X)= & \mathscr{E}_{1}^{e}(X)+\mathscr{E}_{1}^{i}(X)=\mathscr{E}_{1,1}^{e}(X)+\mathscr{E}_{1,2}^{e}(X)+\mathscr{E}_{1}^{i}(X) \\
= & \int_{V_{T}^{e}} \tau_{+}^{2}\left(\left|\hat{\mathscr{L}}_{X} \Psi_{1}\right|\left|J_{-1}^{\prime}(X)\right|+\left|\hat{\mathscr{L}}_{X} \Psi_{0}\right|\left|J_{0}(X)\right|\right) \\
& +\int_{V_{T}^{e}} \tau_{-}^{2}\left(\left|\hat{\mathscr{L}}_{X} \Psi_{-1}\right|\left|J_{-1}(X)\right|+\left|\hat{\mathscr{L}}_{X} \Psi_{0}\right|\left|J_{0}^{\prime}(X)\right|\right) \\
& +\int_{V_{T}^{i}}\left(1+t^{2}\right)\left|\hat{\mathscr{L}}_{X} \Psi\right||J(X)| .
\end{aligned}
$$

Each of the above three integrals are estimated as follows:

1. $\mathscr{E}_{1}^{i}(X), X=\Omega_{i j}, T, S$ : From (70),

$$
\begin{aligned}
\mathscr{E}_{1}^{i}(X) & =\int_{V_{T}^{i}}\left(1+t^{2}\right)\left|\hat{\mathscr{L}}_{X} \Psi\right||J(X)| \\
& \leqq \int_{0}^{T} \int_{\Sigma_{t}^{i}}\left(1+t^{2}\right)^{2}|\nabla \Psi||\Psi|^{2} \\
& \leqq \int_{0}^{T} \frac{\left[Q_{1}^{*}(T)\right]^{1 / 2}[\Psi](T)\left[Q_{0}^{*}(T)\right]^{1 / 2}}{(1+t)^{3 / 2}} d t \\
& \leqq C[\Psi](T)\left[R_{1}^{*}(T) R_{0}^{*}(T)\right]^{1 / 2} .
\end{aligned}
$$

2. $\mathscr{E}_{1}^{e}(\mathcal{O})$ : From $(66),(67)$,

$$
\begin{aligned}
\left|J_{0}(\mathcal{O})\right| & \leqq C r\left|\Psi_{1}\right|\left|\Psi_{0}\right| \\
\left|J_{-1}^{\prime}(\mathcal{O})\right| & \leqq C r\left(\left|\Psi_{1}\right|\left|\Psi_{-1}\right|+\left|\Psi_{0}\right|\left|\Psi_{0}\right|\right),
\end{aligned}
$$




$$
\begin{aligned}
\int_{V_{T}^{e}} r^{3}\left|\hat{\mathscr{L}}_{\mathcal{O}} \Psi_{1}\right|\left|\Psi_{-1}\right|\left|\Psi_{1}\right| & \leqq C \int_{-\infty}^{\infty} d u\left(\int_{C_{u}^{e}} r^{2}\left|\hat{\mathscr{F}}_{\mathcal{O}} \Psi_{1}\right|^{2}\right)^{1 / 2} \sup _{C_{u}^{e}}\left(r\left|\Psi_{-1}\right|\right)\left(\int_{C_{u}^{e}} r^{2}\left|\Psi_{1}\right|^{2}\right)^{1 / 2} \\
& \leqq C \int_{-\infty}^{\infty} \frac{\left[R_{1}^{*}(T)\right]^{1 / 2}[\Psi](T)\left[R_{0}^{*}(T)\right]^{1 / 2}}{(1+|u|)^{3 / 2}} d u \\
& \leqq C[\Psi](T)\left[R_{1}^{*}(T) R_{0}^{*}(T)\right]^{1 / 2}, \\
\int_{V_{T}^{e}} r^{3}\left|\hat{\mathscr{W}}_{\mathcal{O}} \Psi_{1}\right|\left|\Psi_{0}\right|\left|\Psi_{0}\right| & \leqq C \int_{-\infty}^{\infty} d u\left(\int_{C_{u}^{e}} r^{2}\left|\hat{\mathscr{P}}_{\mathcal{O}} \Psi_{1}\right|^{2}\right)^{1 / 2} \sup _{C_{u}^{e}}\left(\frac{r^{2}\left|\Psi_{0}\right|}{\tau_{-}}\right)\left(\int_{C_{u}^{e}} \tau_{-}^{2}\left|\Psi_{0}\right|^{2}\right)^{1 / 2} \\
& \leqq C[\Psi](T)\left[R_{1}^{*}(T) R_{0}^{*}(T)\right]^{1 / 2}, \\
\int_{V_{T}^{e}} r^{3}\left|\hat{\mathscr{W}}_{\mathcal{O}} \Psi_{0}\right|\left|\Psi_{0}\right|\left|\Psi_{1}\right| & \leqq C \int_{-\infty}^{\infty} d u\left(\int_{C_{u}^{e}} \tau_{-}^{2}\left|\hat{\mathscr{L}}_{\mathcal{O}} \Psi_{0}\right|^{2}\right)^{1 / 2} \sup _{C_{u}^{e}}\left(\frac{r^{2}\left|\Psi_{0}\right|}{\tau_{-}}\right)\left(\int_{C_{u}^{e}} \tau^{2}\left|\Psi_{1}\right|^{2}\right)^{1 / 2} \\
& \leqq C[\Psi](T)\left[R_{1}^{*}(T) R_{0}^{*}(T)\right]^{1 / 2},
\end{aligned}
$$

Thus

$$
\mathscr{E}_{1,1}^{e}(\mathcal{O}) \leqq C[\Psi](T)\left[R_{1}^{*}(T) R_{0}^{*}(T)\right]^{1 / 2} .
$$

$\mathscr{E}_{1,2}^{e}$ can be estimated similarly by using another family of light cones $C_{v}^{\prime e}(T)$.

3. $\mathscr{E}_{1}^{e}(S)$ : and $\mathscr{E}_{1}^{e}(T)$ : From (68), (69),

$$
\begin{aligned}
\left|J_{0}(T, \psi)\right|+\left|J_{0}(S, \psi)\right| & \leqq C \tau_{+}\left|\Psi_{1}\right|\left|\Psi_{1}\right|+C \tau_{-}\left(\left|\Psi_{-1}\right|\left|\Psi_{1}\right|+\left|\Psi_{0}\right|\left|\Psi_{0}\right|\right), \\
\left|J_{-1}^{\prime}(T, \psi)\right|+\left|J_{-1}^{\prime}(S, \psi)\right| & \leqq C \tau_{+}\left|\Psi_{0}\right|\left|\Psi_{1}\right|+C \tau_{-}\left|\Psi_{-1}\right|\left|\Psi_{0}\right|
\end{aligned}
$$

We will estimate them term by term as before,

$$
\begin{aligned}
& \int_{V_{T}^{e}} r^{3}\left|\hat{\mathscr{\Psi}}_{S} \Psi_{1}\right|\left|\Psi_{0}\right|\left|\Psi_{1}\right| \\
& \quad \leqq C \int_{-\infty}^{\infty} d u\left(\int_{C_{u}^{e}} r^{2}\left|\hat{\mathscr{S}}_{S} \Psi_{1}\right|^{2}\right)^{1 / 2} \sup _{C_{u}^{e}}\left(r\left|\Psi_{0}\right|\right)\left(\int_{C_{u}^{e}} r^{2}\left|\Psi_{1}\right|^{2}\right)^{1 / 2} \\
& \quad \leqq C[\Psi](T)\left[R_{1}^{*}(T) R_{0}^{*}(T)\right]^{1 / 2}, \\
& \int_{V_{T}^{e}} r^{2} \tau_{-}\left|\hat{\mathscr{W}}_{S} \Psi_{1}\right|\left|\Psi_{-1}\right|\left|\Psi_{0}\right| \\
& \quad \leqq C \int_{-\infty}^{\infty} d u\left(\int_{C_{u}^{e}} r^{2}\left|\hat{\mathscr{S}}_{S} \Psi_{1}\right|^{2}\right)^{1 / 2} \sup _{C_{u}^{e}}\left(r\left|\Psi_{-1}\right|\right)\left(\int_{C_{u}^{e}} \tau_{-}^{2}\left|\Psi_{0}\right|^{2}\right)^{1 / 2} \\
& \leqq C[\Psi](T)\left[R_{1}^{*}(T) R_{0}^{*}(T)\right]^{1 / 2}, \\
& \int_{V_{T}^{e}} r^{3}\left|\hat{\mathscr{P}}_{S} \Psi_{0}\right|\left|\Psi_{1}\right|^{2} \\
& \quad \leqq C \int_{-\infty}^{\infty} d u\left(\int_{C_{u}^{e}} \tau_{-}^{2}\left|\hat{\mathscr{\Psi}}_{S} \Psi_{0}\right|^{2}\right)^{1 / 2} \sup _{C_{u}^{e}}\left(\frac{r^{2}\left|\Psi_{1}\right|}{\tau_{-}}\right)\left(\int_{C_{u}^{e}} \tau^{2}\left|\Psi_{1}\right|^{2}\right)^{1 / 2}
\end{aligned}
$$




$$
\begin{aligned}
& \leqq C[\Psi](T)\left[R_{1}^{*}(T) R_{0}^{*}(T)\right]^{1 / 2}, \\
& \int_{V_{T}^{e}} r^{2} \tau_{-}\left|\hat{\mathscr{P}}_{S} \Psi_{0}\right|\left|\Psi_{-1}\right|\left|\Psi_{1}\right| \\
& \quad \leqq C \int_{-\infty}^{\infty} d u\left(\int_{C_{u}^{e}} \tau_{-}^{2}\left|\hat{\mathscr{F}}_{S} \Psi_{0}\right|^{2}\right)^{1 / 2} \sup _{C_{u}^{e}}\left(r\left|\Psi_{-1}\right|\right)\left(\int_{C_{u}^{e}} r^{2}\left|\Psi_{1}\right|^{2}\right)^{1 / 2} \\
& \quad \leqq C[\Psi](T)\left[R_{1}^{*}(T) R_{0}^{*}(T)\right]^{1 / 2}, \\
& \int_{V_{T}^{e}} r^{2} \tau_{-}\left|\hat{\mathscr{P}}_{S} \Psi_{0}\right|\left|\Psi_{0}\right|^{2} \\
& \leqq C \int_{-\infty}^{\infty} d u\left(\int_{C_{u}^{e}} \tau_{-}^{2}\left|\hat{\mathscr{P}}_{S} \Psi_{0}\right|^{2}\right)^{1 / 2} \sup _{C_{u}^{e}}\left(\frac{r^{2}\left|\Psi_{0}\right|}{\tau_{-}}\right)\left(\int_{C_{u}^{e}} \tau_{-}^{2}\left|\Psi_{0}\right|^{2}\right)^{1 / 2} \\
& \leqq C[\Psi](T)\left[R_{1}^{*}(T) R_{0}^{*}(T)\right]^{1 / 2} .
\end{aligned}
$$

Thus

$$
\mathscr{E}_{1,1}^{e}(S) \leqq C[\Psi](T)\left[R_{1}^{*}(T) R_{0}^{*}(T)\right]^{1 / 2}
$$

We can similarly estimate $\mathscr{E}_{1,2}^{e}(S)$ and $\mathscr{E}_{1}^{e}(T)$. In conclusion, we obtain the following estimate:

$$
Q_{1}^{*}(T) \leqq C Q_{1}(0)+C[\Psi](T)\left[R_{1}^{*}(T) R_{2}^{*}(T)\right]^{1 / 2} .
$$

From the norms of equivalence in Step 1,

$$
Q_{1}^{*}(T) \leqq C Q_{1}(0)+C \varepsilon_{1} Q_{1}^{*}(T) .
$$

Therefore we proved Step 2 by choosing $\varepsilon_{1}$ small, s.t. $c \varepsilon_{1}<\frac{1}{2}$.

Step 3. Estimate of $Q_{2}^{*}(T)$ : if $\varepsilon_{1}$ is sufficiently small, then

$$
Q_{2}^{*}(T) \leqq C Q_{2}(0) \text {. }
$$

The proof is very similar to that in Step 2. Apply Lemma 10 for $\mathscr{L}_{X} \mathscr{L}_{Y} \Psi_{A B}$, where $X, Y=\Omega_{i j}, S, T$, we have to estimate the following error terms:

$$
\begin{aligned}
\mathscr{E}_{2}(X, Y)= & \mathscr{E}_{2}^{e}(X, Y)+\mathscr{E}_{2}^{i}(X, Y)=\mathscr{E}_{2,1}^{e}(X, Y)+\mathscr{E}_{2,2}^{e}(X, Y)+\mathscr{E}_{2}^{i}(X, Y) \\
= & \int_{V_{T}^{e}} \tau_{+}^{2}\left(\left|\hat{\mathscr{L}}_{X} \hat{\mathscr{P}}_{Y} \Psi_{1}\right|\left|J_{-1}^{\prime}(X, Y)\right|+\left|\hat{\mathscr{L}}_{X} \hat{\mathscr{P}}_{Y} \Psi_{0}\right|\left|J_{0}(X, Y)\right|\right) \\
& +\int_{V_{T}^{e}} \tau_{-}^{2}\left(\left|\hat{\mathscr{L}}_{X} \hat{\mathscr{P}}_{Y} \Psi_{-1}\right|\left|J_{-1}(X, Y)\right|+\left|\hat{\mathscr{L}}_{X} \hat{\mathscr{P}}_{Y} \Psi_{0}\right|\left|J_{0}^{\prime}(X, Y)\right|\right) \\
& +\int_{V_{T}^{i}}\left(1+t^{2}\right)\left|\hat{\mathscr{L}}_{X} \hat{\mathscr{L}}_{Y} \Psi\right||J(X, Y)| .
\end{aligned}
$$

The interior part is obvious,

$$
\begin{aligned}
\mathscr{E}_{2}^{i}(X, Y) & \leqq \int_{0}^{T} \int_{\Sigma_{t}^{2}}\left(1+t^{2}\right)^{4}\left|\nabla^{2} \Psi\right||\Psi||\nabla \Psi| \\
& \leqq C[\Psi](T)\left[R_{1}^{*}(T) R_{2}^{*}(T)\right]^{1 / 2}
\end{aligned}
$$


The exterior parts are estimated term by term as follows:

1. $\mathscr{E}_{2}^{e}(\mathcal{O}, \mathcal{O})$ : Applying Lemma 10 with $\psi_{A B}=\hat{\mathscr{L}}_{Y} \Psi_{A B}$, for $X, Y \in \mathcal{O}$,

$$
\begin{aligned}
\left|J_{0}(\mathcal{O}, \mathcal{O})\right| & \leqq C r\left(\left|\Psi_{1}\right|\left|\hat{\mathscr{L}}_{\mathcal{O}} \Psi_{0}\right|+\left|\Psi_{0}\right|\left|\hat{\mathscr{L}}_{\mathcal{O}} \Psi_{1}\right|\right) \\
\left|J_{-1}^{\prime}(\mathcal{O}, \mathcal{O})\right| & \leqq C r\left(\left|\Psi_{1}\right|\left|\hat{\mathscr{L}}_{\mathcal{O}} \Psi_{-1}\right|+\left|\Psi_{-1}\right|\left|\hat{\mathscr{P}}_{\mathcal{O}} \Psi_{1}\right|+\left|\Psi_{0}\right|\left|\hat{\mathscr{L}}_{\mathcal{O}} \Psi_{0}\right|\right) .
\end{aligned}
$$

Thus

$$
\begin{aligned}
& \int_{V_{T}^{e}} r^{3}\left|\hat{\mathscr{L}}_{\mathcal{O}} \hat{\mathscr{F}}_{\mathcal{O}} \Psi_{1}\right|\left|\Psi_{-1}\right|\left|\hat{\mathscr{F}}_{\mathcal{O}} \Psi_{1}\right| \\
& \quad \leqq C \int_{-\infty}^{\infty} d u\left(\int_{C_{u}^{e}} r^{2}\left|\hat{\mathscr{L}}_{\mathcal{O}} \hat{\mathscr{L}}_{\mathcal{O}} \Psi_{1}\right|^{2}\right)^{1 / 2} \sup _{C_{u}^{e}}\left(r\left|\Psi_{-1}\right|\right)\left(\int_{C_{u}^{e}} r^{2}\left|\hat{\mathscr{L}}_{\mathcal{O}} \Psi_{1}\right|^{2}\right)^{1 / 2} \\
& \quad \leqq C[\Psi](T)\left[R_{2}^{*}(T) R_{1}^{*}(T)\right]^{1 / 2},
\end{aligned}
$$

To estimate

$$
\int_{V_{T}^{e}} r^{3}\left|\hat{\mathscr{L}}_{\mathcal{O}} \hat{\mathscr{L}}_{\mathcal{O}} \Psi_{1}\right|\left|\Psi_{1}\right|\left|\hat{\mathscr{L}}_{\mathcal{O}} \Psi_{-1}\right|
$$

we need to be a little more careful because the energy estimate does not give us any bound on the integral of $\hat{\mathscr{L}}_{\mathcal{O}} \Psi_{-1}$ over $C_{u}(T)$,

$$
\begin{aligned}
& \int_{V_{T}^{e}} r^{3}\left|\hat{\mathscr{L}}_{\mathcal{O}} \hat{\mathscr{L}}_{\mathcal{O}} \Psi_{1}\right|\left|\Psi_{1}\right|\left|\hat{\mathscr{L}}_{\mathcal{O}} \Psi_{-1}\right| \\
& \leqq \int_{-\infty}^{\infty} d u\left(\int_{C_{u}^{e}} r^{2}\left|\hat{\mathscr{L}}_{\mathcal{O}} \hat{\mathscr{L}}_{\mathcal{O}} \Psi_{1}\right|^{2}\right)^{1 / 2} \\
& \cdot\left[\int_{r_{m}(u)}^{r_{M}(u)} d r\left(\int_{\tilde{S}_{u, r}} r^{2}\left|\hat{\mathscr{P}}_{\mathcal{O}} \Psi_{-1}\right|^{4}\right)^{1 / 2}\left(\int_{\tilde{S}_{u, r}} r^{6}\left|\Psi_{1}\right|^{4}\right)^{1 / 2}\right]^{1 / 2} \\
& \leqq \int_{-\infty}^{\infty} d u\left(\int_{C_{u}^{e}} r^{2}\left|\hat{\mathscr{P}}_{\mathcal{O}} \hat{\mathscr{P}}_{\mathcal{O}} \Psi_{1}\right|^{2}\right)^{1 / 2} \frac{\sup _{r_{m}(u)<r<r_{M}(u)}\left(\int_{\tilde{S}_{u, r}} r^{2} \tau_{-}^{6}\left|\hat{\mathscr{P}}_{\mathcal{O}} \Psi_{-1}\right|^{4}\right)^{1 / 4}}{\tau_{-}^{3 / 2}} \\
& \cdot\left[\int_{r_{m}(u)}^{r_{M}(u)} d r\left(\int_{\tilde{S}_{u, r}} r^{6}\left|\Psi_{1}\right|^{4}\right)^{1 / 2}\right]^{1 / 2} .
\end{aligned}
$$

Apply (54) of the Lemma 7 for $f=\tau_{-}\left|\hat{\mathscr{A}}_{\mathcal{O}} \Psi_{-1}\right|$ to obtain

$$
\left(\int_{\tilde{S}_{u, r}} r^{2} \tau_{-}^{6}\left|\hat{\mathscr{P}}_{\mathcal{O}} \Psi_{-1}\right|^{4}\right)^{1 / 2} \leqq R_{2}^{*}(T) .
$$

Applying to $f=r^{3}\left|\Psi_{1}\right|^{2}$ the Isoperimetric inequality (52) and then integrate from $r_{m}$ to $r_{M}$,

$$
\begin{aligned}
\int_{r_{m}(u)}^{r_{M}(u)} d r\left(\int_{\tilde{S}_{u, r}} r^{6}\left|\Psi_{1}\right|^{4}\right)^{1 / 2} & \leqq \int_{r_{m}(u)}^{r_{M}(u)} d r \int_{\tilde{S}_{u, r}}\left(r^{2}\left|\Psi_{1}\right|^{2}+3 r^{2}\left|\Psi_{1}\right|^{2}+2 r^{3}\left|\Psi_{1}\right||\nabla \Psi|\right) \\
& \leqq C \int_{C_{u}^{e}(T)} r^{2}|\Psi|^{2}+r^{4}|\nabla \Psi|^{2} \leqq C R_{1}^{*}(T) .
\end{aligned}
$$


Therefore we deduce

$$
\int_{V_{T}^{e}} r^{3}\left|\hat{\mathscr{P}}_{\mathcal{O}} \hat{\mathscr{P}}_{\mathcal{O}} \Psi_{1}\right|\left|\Psi_{1}\right|\left|\hat{\mathscr{P}}_{\mathcal{O}} \Psi_{-1}\right| \leqq C R_{2}^{*}(T)\left[R_{1}^{*}(T)\right]^{1 / 2}
$$

Next,

$$
\begin{aligned}
& \int_{V_{T}^{e}} r^{3}\left|\hat{\mathscr{L}}_{\mathcal{O}} \hat{\mathscr{L}}_{\mathcal{O}} \Psi_{0}\right|\left|\Psi_{0}\right|\left|\hat{\mathscr{L}}_{\mathcal{O}} \Psi_{-1}\right| \\
& \quad \leqq C \int_{-\infty}^{\infty} d u\left(\int_{C_{u}^{e}(T)} \tau_{-}^{2}\left|\hat{\mathscr{L}}_{\mathcal{O}} \hat{\mathscr{P}}_{\mathcal{O}} \Psi_{0}\right|^{2}\right)^{1 / 2} \sup _{C_{u}^{e}}\left(\frac{r^{2}\left|\Psi_{0}\right|}{\tau_{-}}\right)\left(\int_{C_{u}^{e}} r^{2}\left|\hat{\mathscr{L}}_{\mathcal{O}} \Psi_{1}\right|^{2}\right)^{1 / 2} \\
& \quad \leqq C[\Psi](T)\left[R_{2}^{*}(T) R_{1}^{*}(T)\right]^{1 / 2} . \\
& \int_{V_{T}^{e}} r^{3}\left|\hat{\mathscr{P}}_{\mathcal{O}} \hat{\mathscr{P}}_{\mathcal{O}} \Psi_{0}\right|\left|\Psi_{1}\right|\left|\hat{\mathscr{P}}_{\mathcal{O}} \Psi_{0}\right| \\
& \quad \leqq C \int_{0}^{\infty} d v\left(\int_{C_{v}^{\prime} e_{(T)}} r^{2}\left|\hat{\mathscr{P}}_{\mathcal{O}} \hat{\mathscr{P}}_{\mathcal{O}} \Psi_{0}\right|^{2}\right)^{1 / 2} \sup _{C_{v}^{\prime e}(T)}\left(r\left|\Psi_{1}\right|\right)\left(\int_{C_{v}^{\prime}(T)} r^{2}\left|\hat{\mathscr{P}}_{\mathcal{O}} \Psi_{0}\right|^{2}\right) \\
& \quad \leqq C[\Psi](T)\left[R_{2}^{*}(T) R_{1}^{*}(T)\right]^{1 / 2} .
\end{aligned}
$$

Thus

$$
\mathscr{E}_{2,1}^{e}(\mathcal{O}, \mathcal{O}) \leqq C[\Psi](T)\left[R_{2}^{*}(T) R_{1}^{*}(T)\right]^{1 / 2}+C R_{2}^{*}(T)\left[R_{1}^{*}(T)\right]^{1 / 2}
$$

Similarly we can estimate $\mathscr{E}_{2,2}(\mathcal{O}, \mathcal{O})$.

2. $\mathscr{E}_{2}^{e}(S, \mathcal{O}), \mathscr{E}_{2}^{e}(T, \mathcal{O}), \mathscr{E}_{2}^{e}(\mathcal{O}, S)$ and $\mathscr{E}_{2}^{e}(\mathcal{O}, T)$ : The proofs are all very similar. For example, we will estimate $\mathscr{E}_{2}^{e}(S, \mathcal{O})$ as follows,

$$
\begin{aligned}
& \left|J_{0}(S, \mathcal{O})\right| \leqq C \tau_{+}\left|\Psi_{1}\right|\left|\hat{\mathscr{L}}_{\mathcal{O}} \Psi_{1}\right| \\
& +C \tau_{-}\left(\left|\Psi_{-1}\right|\left|\hat{\mathscr{A}}_{\mathcal{O}} \Psi_{1}\right|+\left|\Psi_{1}\right|\left|\hat{\mathscr{A}}_{\mathcal{O}} \Psi_{-1}\right|+\left|\Psi_{0}\right|\left|\hat{\mathscr{A}}_{\mathcal{O}} \Psi_{0}\right|\right) \\
& +\operatorname{Cr}\left(\left|\hat{\mathscr{L}}_{S} \Psi_{1}\right|\left|\Psi_{0}\right|+\left|\hat{\mathscr{L}}_{S} \Psi_{0}\right|\left|\Psi_{1}\right|\right) \text {, } \\
& \left|J_{-1}^{\prime}(S, \mathcal{O})\right| \leqq C \tau_{+}\left(\left|\Psi_{0}\right|\left|\hat{\mathscr{L}}_{\mathcal{O}} \Psi_{1}\right|+\left|\Psi_{1}\right|\left|\hat{\mathscr{L}}_{\mathcal{O}} \Psi_{0}\right|\right) \\
& +C \tau_{-}\left(\left|\Psi_{-1}\right|\left|\hat{\mathscr{A}}_{\mathcal{O}} \Psi_{0}\right|+\left|\Psi_{1}\right|\left|\hat{\mathscr{P}}_{\mathcal{O}} \Psi_{-1}\right|\right) \\
& +\operatorname{Cr}\left(\left|\hat{\mathscr{L}}_{S} \Psi_{1}\right|\left|\Psi_{-1}\right|+\left|\hat{\mathscr{L}}_{S} \Psi_{-1}\right|\left|\Psi_{1}\right|+\left|\hat{\mathscr{L}}_{S} \Psi_{0}\right|\left|\Psi_{0}\right|\right)
\end{aligned}
$$

Thus

$$
\begin{aligned}
& \int_{V_{T}^{e}} r^{3}\left|\hat{\mathscr{P}}_{\mathcal{O}} \hat{\mathscr{P}}_{S} \Psi_{1}\right|\left|\Psi_{0}\right|\left|\hat{\mathscr{P}}_{\mathcal{O}} \Psi_{1}\right| \\
& \leqq C \int_{-\infty}^{\infty} d u\left(\int_{C_{u}^{e}} r^{2}\left|\hat{\mathscr{L}}_{\mathcal{O}} \hat{\mathscr{L}}_{S} \Psi_{1}\right|^{2}\right)^{1 / 2} \sup _{C_{u}^{e}(T)}\left(r\left|\Psi_{0}\right|\right)\left(\int_{C_{u}^{e}(T)} r^{2}\left|\hat{\mathscr{L}}_{\mathcal{O}} \Psi_{1}\right|^{2}\right)^{1 / 2} \\
& \leqq C[\Psi](T)\left[R_{2}^{*}(T) R_{1}^{*}(T)\right]^{1 / 2} . \\
& \int_{V_{T}^{e}} r^{3}\left|\hat{\mathscr{L}}_{\mathcal{O}} \hat{\mathscr{L}}_{S} \Psi_{1}\right|\left|\Psi_{1}\right|\left|\Psi_{1}\right|\left|\hat{\mathscr{L}}_{\mathcal{O}} \Psi_{0}\right| \\
& \leqq C \int_{-\infty}^{\infty} d u\left(\int_{C_{u}^{e}} r^{2}\left|\hat{\mathscr{P}}_{\mathcal{O}} \hat{\mathscr{P}}_{S} \Psi_{1}\right|^{2}\right)^{1 / 2} \sup _{C_{u}^{e}(T)}\left(\frac{r^{2}\left|\Psi_{1}\right|}{\tau_{-}}\right)\left(\int_{C_{u}^{e}(T)} \tau_{-}^{2}\left|\hat{\mathscr{P}}_{\mathcal{O}} \Psi_{0}\right|^{2}\right)^{1 / 2} \\
& \leqq C[\Psi](T)\left[R_{2}^{*}(T) R_{1}^{*}(T)\right]^{1 / 2} \text {. }
\end{aligned}
$$




$$
\begin{aligned}
& \int_{V_{T}^{e}} r^{3}\left|\hat{\mathscr{L}}_{\mathcal{O}} \hat{\mathscr{L}}_{S} \Psi_{1}\right|\left|\hat{\mathscr{L}}_{S} \Psi_{1}\right|\left|\Psi_{-1}\right| \\
& \leqq C \int_{-\infty}^{\infty} d u\left(\int_{C_{u}^{e}} r^{2}\left|\hat{\mathscr{L}}_{\mathcal{O}} \hat{\mathscr{L}}_{S} \Psi_{1}\right|^{2}\right)^{1 / 2} \sup _{C_{u}^{e}(T)}\left(r^{2}\left|\Psi_{-1}\right|\right)\left(\int_{C_{u}^{e}(T)} r^{2}\left|\hat{\mathscr{L}}_{S} \Psi_{1}\right|^{2}\right)^{1 / 2} \\
& \leqq C[\Psi](T)\left[R_{2}^{*}(T) R_{1}^{*}(T)\right]^{1 / 2} . \\
& \int_{V_{T}^{e}} r^{3}\left|\mathscr{L}_{O} \hat{\mathscr{P}}_{S} \Psi_{1}\right|\left|\hat{\mathscr{L}}_{S} \Psi_{-1}\right| \mid \Psi_{1} \\
& \leqq C \int_{-\infty}^{\infty} d u\left(\int_{C_{u}^{e}} r^{2}\left|\hat{\mathscr{L}}_{\mathcal{O}} \hat{\mathscr{P}}_{\mathrm{S}} \Psi_{1}\right|^{2}\right)^{1 / 2} \\
& \cdot\left\{\int_{r_{e}(u)}^{r_{e}(T)} d r\left(\int_{\tilde{S}_{u, r}} r^{2}\left|\hat{\mathscr{P}}_{S} \Psi_{-1}\right|^{4}\right)^{1 / 2}\left(\int_{\tilde{S}_{u, r}} r^{6}\left|\Psi_{1}\right|^{4}\right)^{1 / 2}\right\}^{1 / 2}
\end{aligned}
$$

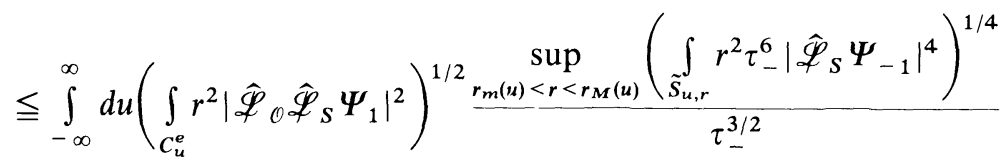

$$
\begin{aligned}
& \left\{\int_{r_{m}(u)}^{r_{M}(u)} d r\left(\int_{\tilde{S}_{u, r}} r^{6}\left|\Psi_{1}\right|^{4}\right)^{1 / 2}\right\}^{1 / 2} \\
& \leqq C R_{2}^{*}(T)\left[R_{1}^{*}(T)\right]^{1 / 2},
\end{aligned}
$$

where in the last inequality, we use the similar reason as that in (78) and (79).

$$
\begin{aligned}
& \int_{V_{T}^{e}} r^{3}\left|\hat{\mathscr{F}}_{O} \hat{\mathscr{L}}_{S} \Psi_{1}\right|\left|\hat{\mathscr{L}}_{S} \Psi_{0}\right|\left|\Psi_{0}\right| \\
& \leqq C \int_{-\infty}^{\infty} d u\left(\int_{C_{u}^{e}} r^{2}\left|\hat{\mathscr{A}}_{\mathcal{O}} \hat{\mathscr{L}}_{S} \Psi_{0}\right|^{2}\right)^{1 / 2} \sup _{C_{u}^{e}(T)}\left(\frac{r^{2}\left|\Psi_{0}\right|}{\tau_{-}}\right)\left(\int_{C_{u}^{e}(T)} r_{-}^{2}\left|\hat{\mathscr{L}}_{S} \Psi_{0}\right|^{2}\right)^{1 / 2} \\
& \leqq C[\Psi](T)\left[R_{2}^{*}(T) R_{1}^{*}(T)\right]^{1 / 2}, \\
& \int_{V_{T}^{e}} r^{2} \tau_{-}\left|\hat{\mathscr{L}}_{\mathcal{O}} \hat{\mathscr{F}}_{S} \Psi_{1}\right|\left|\Psi_{-1}\right|\left|\hat{\mathscr{F}}_{\mathcal{O}} \Psi_{0}\right| \\
& \leqq C \int_{-\infty}^{\infty} d u\left(\int_{C_{u}^{e}} r^{2}\left|\hat{\mathscr{A}}_{\mathcal{O}} \hat{\mathscr{L}}_{S} \Psi_{0}\right|^{2}\right)^{1 / 2} \sup _{C_{u}^{e}(T)}\left(r\left|\Psi_{-1}\right|\right)\left(\int_{C_{u}^{e}(T)} \tau_{-}^{2}\left|\hat{\mathscr{L}}_{\mathcal{O}} \Psi_{0}\right|^{2}\right)^{1 / 2} \\
& \leqq C[\Psi](T)\left[R_{2}^{*}(T) R_{1}^{*}(T)\right]^{1 / 2}, \\
& \int_{V_{T}^{e}} r^{2} \tau_{-}\left|\hat{\mathscr{L}}_{\mathcal{O}} \hat{\mathscr{P}}_{S} \Psi_{1}\right|\left|\Psi_{0}\right|\left|\hat{\mathscr{L}}_{\mathcal{O}} \Psi_{-1}\right| \\
& \leqq C \int_{-\infty}^{\infty} d u\left(\int_{C_{u}^{e}} r^{2}\left|\hat{\mathscr{L}}_{\mathcal{O}} \hat{\mathscr{F}}_{S} \Psi_{0}\right|^{2}\right)^{1 / 2} \\
& \cdot\left\{\int_{r_{m}(u)}^{r_{M}^{(u)}} d r\left(\int_{\tilde{S}_{u, r}} r^{2}\left|\hat{\mathscr{P}}_{S} \Psi_{-1}\right|^{4}\right)^{1 / 2}\left(\int_{\tilde{S}_{u, r}} r^{2} \tau_{-}^{4}\left|\Psi_{0}\right|^{4}\right)^{1 / 2}\right\}^{1 / 2} \\
& \leqq C R_{2}^{*}(T)\left[R_{1}^{*}(T)\right]^{1 / 2},
\end{aligned}
$$


where in the last step, we use the same trick as that in (78) and (79).

$$
\begin{aligned}
& \int_{V_{T}^{e}} r^{3}\left|\hat{\mathscr{P}}_{\mathcal{O}} \hat{\mathscr{P}}_{S} \Psi_{0}\right|\left|\Psi_{1}\right|\left|\hat{\mathscr{L}}_{\mathcal{O}} \Psi_{1}\right| \\
& \leqq C \int_{-\infty}^{\infty} d u\left(\int_{C_{u}^{e}} \tau_{-}^{2}\left|\hat{\mathscr{P}}_{\mathcal{O}} \hat{\mathscr{F}}_{S} \Psi_{0}\right|^{2}\right)^{1 / 2} \sup _{C_{u}^{e}(T)}\left(\frac{r^{2}\left|\Psi_{1}\right|}{\tau_{-}}\right)\left(\int_{C_{u}^{e}(T)} r^{2}\left|\hat{\mathscr{F}}_{\mathcal{O}} \Psi_{1}\right|^{2}\right)^{1 / 2} \\
& \leqq C[\Psi](T)\left[R_{2}^{*}(T) R_{1}^{*}(T)\right]^{1 / 2}, \\
& \int_{V_{T}^{e}} r^{2} \tau_{-}\left|\hat{\mathscr{L}}_{\mathcal{O}} \hat{\mathscr{L}}_{S} \Psi_{0}\right|\left|\Psi_{-1}\right|\left|\hat{\mathscr{L}}_{\mathcal{O}} \Psi_{1}\right| \\
& \leqq C \int_{-\infty}^{\infty} d u\left(\int_{C_{u}^{e}} \tau_{-}^{2}\left|\hat{\mathscr{L}}_{\mathcal{O}} \hat{\mathscr{L}}_{S} \Psi_{0}\right|^{2}\right)^{1 / 2} \sup _{C_{u}^{e}(T)}\left(r\left|\Psi_{-1}\right|\right)\left(\int_{C_{u}^{e}(T)} r^{2}\left|\hat{\mathscr{L}}_{\mathcal{O}} \Psi_{1}\right|^{2}\right)^{1 / 2} \\
& \leqq C[\Psi](T)\left[R_{2}^{*}(T) R_{1}^{*}(T)\right]^{1 / 2}, \\
& \int_{V_{T}^{e}} r^{2} \tau_{-}\left|\hat{\mathscr{P}}_{\mathcal{O}} \hat{\mathscr{P}}_{S} \Psi_{0}\right|\left|\Psi_{1}\right|\left|\hat{\mathscr{P}}_{\mathcal{O}} \Psi_{-1}\right| \\
& \leqq C \int_{0<v<\infty}\left(\int_{C_{v}^{\prime}(T)} r^{2}\left|\hat{\mathscr{L}}_{\mathcal{O}} \hat{\mathscr{L}}_{S} \Psi_{0}\right|^{2}\right)^{1 / 2} \sup _{C_{v}^{\prime e}(T)}\left(r\left|\Psi_{1}\right|\right)\left(\int_{C_{v}^{\prime e}(T)} \tau_{-}^{2}\left|\hat{\mathscr{L}}_{\mathcal{O}} \Psi_{-1}\right|^{2}\right)^{1 / 2} \\
& \leqq C[\Psi](T)\left[R_{2}^{*}(T) R_{1}^{*}(T)\right]^{1 / 2}, \\
& \int_{V_{T}^{e}} r^{3}\left|\hat{\mathscr{L}}_{\mathcal{O}} \hat{\mathscr{L}}_{S} \Psi_{0}\right|\left|\hat{\mathscr{A}}_{S} \Psi_{1}\right|\left|\Psi_{0}\right| \\
& \leqq C \int_{-\infty}^{\infty} d u\left(\int_{C_{u}^{e}} \tau_{-}^{2}\left|\hat{\mathscr{L}}_{O} \hat{\mathscr{L}}_{S} \Psi_{0}\right|^{2}\right)^{1 / 2} \sup _{C_{u}^{e}(T)}\left(\frac{r^{2}\left|\Psi_{d}\right|}{\tau_{-}}\right)\left(\int_{C_{u}^{e}(T)} r^{2}\left|\hat{\mathscr{F}}_{S} \Psi_{1}\right|^{2}\right)^{1 / 2} \\
& \leqq C[\Psi](T)\left[R_{2}^{*}(T) R_{1}^{*}(T)\right]^{1 / 2}, \\
& \int_{V_{T}^{e}} r^{3}\left|\hat{\mathscr{L}}_{\mathcal{O}} \hat{\mathscr{P}}_{S} \Psi_{0}\right|\left|\hat{\mathscr{L}}_{S} \Psi_{0}\right|\left|\Psi_{1}\right| \\
& \leqq C \int_{0<v<\infty}\left(\int_{C_{v}^{\prime e}(T)} r^{2}\left|\hat{\mathscr{L}}_{\mathcal{O}} \hat{\mathscr{P}}_{S} \Psi_{0}\right|^{2}\right)^{1 / 2} \sup _{C_{v}^{\prime}(T)}\left(r\left|\Psi_{1}\right|\right)\left(\int_{C_{v}^{\prime}(T)} r^{2}\left|\hat{\mathscr{L}}_{\mathcal{O}} \Psi_{0}\right|^{2}\right)^{1 / 2} \\
& \leqq C[\Psi](T)\left[R_{2}^{*}(T) R_{1}^{*}(T)\right]^{1 / 2} .
\end{aligned}
$$

Therefore we have the following estimate

$$
\mathscr{E}_{2,1}^{e}(S, \mathcal{O}) \leqq C[\Psi](T)\left[R_{2}^{*}(T) R_{1}^{*}(T)\right]^{1 / 2}+C R_{2}^{*}(T)\left[R_{1}^{*}(T)\right]^{1 / 2}
$$

$\mathscr{E}_{2,2}^{e}(S, \mathcal{O})$ can be estimated in the same way.

3. $\mathscr{E}_{2}^{e}(S, S)$ :

$$
\begin{aligned}
\left|J_{0}(S, S)\right| \leqq & C \tau_{+}\left(\left|\Psi_{1}\right|\left|\hat{\mathscr{L}}_{S} \Psi_{1}\right|\left|\Psi_{1}\right|\left|\Psi_{1}\right|\right) \\
& +C \tau_{-}\left(\left|\Psi_{-1}\right|\left|\hat{\mathscr{L}}_{S} \Psi_{1}\right|+\left|\Psi_{1}\right|\left|\hat{\mathscr{P}}_{S} \Psi_{-1}\right|+\left|\Psi_{0}\right|\left|\hat{\mathscr{L}}_{S} \Psi_{0}\right|\right) \\
& +C \tau_{-}\left(\left|\Psi_{-1}\right|\left|\Psi_{1}\right|+\left|\Psi_{0}\right|\left|\Psi_{0}\right|\right)
\end{aligned}
$$




$$
\begin{aligned}
\left|J_{1}(S, S)\right| \leqq & C \tau_{+}\left(\left|\Psi_{0}\right|\left|\hat{\mathscr{P}}_{S} \Psi_{1}\right|+\left|\Psi_{1}\right|\left|\hat{\mathscr{P}}_{S} \Psi_{0}\right|+\left|\Psi_{0}\right|\left|\Psi_{1}\right|\right) \\
& +C \tau_{-}\left(\left|\Psi_{-1}\right|\left|\hat{\mathscr{P}}_{S} \Psi_{0}\right|+\left|\Psi_{0}\right|\left|\hat{\mathscr{P}}_{S} \Psi_{-1}\right|+\left|\Psi_{-1}\right| \mid \Psi_{0}\right)
\end{aligned}
$$

The estimates for these terms are essentially the same as before, so we will omit them.

Summing up, we have proved the claim. This completes the proof of Theorem 3.

Now let's investigate how the presence of charge on the initial data will affect the asymptotic behavior of the solution. I conjecture that this long range effect of the initial data will only change the asymptotic behavior at the space like infinity, it will not change the asymptotic behavior of the solution along the time-like and null infinity. I can only verify this statement under the assumption that the initial data is Abelian outside a compact set, say $\{r \leqq 1\}$, that is if

$$
\phi_{i}(0, x)=\varphi_{i}(x) \sigma, \quad E_{i}(0, x)=f_{i}(x) \sigma, \quad r=|x| \geqq 1,
$$

where $\sigma \in \mathscr{G}$ is fixed, $\varphi_{i}(x)$ and $f_{i}(x)$ are two real functions. For this type of initial data the charges are defined as follows:

$$
\begin{aligned}
& e=\frac{1}{4 \pi} \int_{S_{\infty}} \frac{x^{i}}{r} E_{i}(0, x)=\frac{1}{4 \pi} \lim _{r \rightarrow \infty} \int_{S_{r}} \frac{x^{i}}{r} E_{i}, \\
& q=\frac{1}{4 \pi} \int_{S_{\infty}} \frac{x^{i}}{r} H_{i}(0, x)=\frac{1}{4 \pi} \lim _{r \rightarrow \infty} \int_{S_{r}} \frac{x^{i}}{r} H_{i},
\end{aligned}
$$

$e$ and $q$ are respectively called the electric and magnetic charge. For the general case, the first question to ask is how to give a gauge independent definition of the charges. I am not aware of any answer to this question.

Lemma 12. For any spin-s weighted scalar $\psi$,

$$
\begin{array}{r}
\int_{S_{t, r}} r^{2}|\nabla \psi|^{2}+|\psi|^{2} \leqq C \int_{S_{t, r}}\left|\hat{\mathscr{L}}_{\mathcal{O}} \psi\right|^{2}, \quad s \neq 0, \\
\int_{S_{t, r}} r^{2}|\nabla \psi|^{2}+|\psi-\operatorname{Ave}(\psi)|^{2} \leqq C \int_{S_{t, r}}\left|\hat{\mathscr{F}}_{\mathcal{O}} \psi\right|^{2}, \quad s=0,
\end{array}
$$

where Ave $(\psi)$ is the average of $\psi$ over $S_{t, r}$.

For spin- 0 weighted scalar $\psi$ the Lie derivative $\hat{\mathscr{P}}_{\mathcal{O}} \psi$ is just the usual derivative, therefore (84) is just the usual Poincare inequality; (83) is a result of direct calculation (for the tensor version see [3]).

Theorem 4. Suppose the initial data is smooth, Abelian outside a compact set, say $\{r \leqq 1\}$, and satisfies the constraint equation (60). Assume

$$
\left\|\mathscr{L}_{\mathcal{O}} \Psi(0, x)\right\|_{H_{2,1}}+|e(0)|+|q(0)| \leqq \varepsilon_{0},
$$

where $\varepsilon_{0}$ is a small constant. Then there exists a unique global solution of Yang-Mills equations; moreover the curvature spinor has the following decay estimate:

$$
\begin{aligned}
\left|\Psi_{1}(t, x)\right| & \leqq C \varepsilon_{0} \tau_{+}^{-5 / 2} \\
\left|\Psi_{-1}(t, x)\right| & \leqq C \varepsilon_{0} \tau_{+}^{-1} \tau_{-}^{-3 / 2},
\end{aligned}
$$




$$
\mid \Psi_{0}(t, x) \leqq\left\{\begin{array}{ll}
C \varepsilon_{0} \tau_{+}^{-2} \tau_{-}^{-1 / 2}, & \text { if } \quad r<1+t \\
C \varepsilon_{0} \tau_{+}^{-2}, & \text { if } \quad r>1+t
\end{array} .\right.
$$

Proof. Because the initial data is Abelian when $r \geqq 1$, from Hygence principle, we know when $r \geqq 1+t, \Psi_{A B}(t, x)=\psi_{A B}(t, x) \sigma$, where $\psi_{A B}$ is a usual spin-1 field which satisfies the usual spin-1 equation. Therefore the same proof as Theorem 1 in Sect. 4 yields the following estimates,

$$
\begin{gathered}
\tilde{Q}_{2}\left(\mathscr{L}_{\mathcal{O}} \psi\right)(u) \leqq C \varepsilon_{0}^{2}, \quad u \leqq-1, \\
Q_{2}^{\text {out }}\left(\mathscr{L}_{\mathcal{O}} \psi\right)(t) \leqq C \varepsilon_{0}^{2}, \quad \tilde{Q}_{2}^{\text {out }}\left(\mathscr{L}_{\mathcal{O}} \psi\right)(v) \leqq C \varepsilon_{0}^{2},
\end{gathered}
$$

where $Q_{s}^{\text {out }}(T)$ and $\tilde{Q}_{s}^{\text {out }}(v, t)$ are the similar energy norms as $Q_{s}(t)$ and $\tilde{Q}_{s}^{\prime}(v, T)$ except now we only integrate over the corresponding regions intersecting with $r \geqq 1+t$.

We integrate (33), (33)' over $S_{t, r}(r \geqq t+1)$ to deduce

$$
\begin{aligned}
& \left(\partial_{t}+\partial_{r}+\frac{2}{r}\right) \operatorname{Ave}(\psi)=0 \\
& \left(\partial_{t}-\partial_{r}-\frac{2}{r}\right) \operatorname{Ave}(\psi)=0 ;
\end{aligned}
$$

therefore

$$
\operatorname{Ave}(\psi)=\frac{1}{\sqrt{2} r^{2}}(e+i q) \sigma
$$

Thus from Lemma 12 and the assumption (85), we obtain

$$
\tilde{Q}_{2}(1)=\sup _{0<T<\infty} \tilde{Q}_{2}(1, T) \leqq C \varepsilon_{0}^{2}
$$

The rest of the proof is the same as that of Theorem 3 except we always restrict ourself to the region $\{r \leqq 1+t\}$.

Finally I make the following remark:

Remark. The same proof works as well to prove the global existence for the solutions of the initial value problem of the Yang-Mills equations in Schwartzchild space-time outside the black hole. The only problem is that we no longer have the conformal Killing vector fields $S$ and $\bar{K}_{0}$, but we can use the following two asymptotically conformal Killing vector fields,

$$
S=t \partial_{t}+r_{*} \partial_{r_{*}} \quad \bar{K}_{0}=\left(1+t^{2}+r_{*}^{2}\right) \partial_{t}+2 \operatorname{tr}_{*} \partial_{r_{*}}
$$

where $r_{*}=r+2 m \ln (r-2 m)$. The details were given in [13]. I also remark that by combining with the work of Christodoulou and Klainerman in [4], one should also get the global existence of the solutions of the Einstein-Yang-Mills equations. Of course, the proof of such a result will also be very long.

Acknowledgements. Part of this paper is my Ph.D. thesis; I am greatly indebted to my advisor Professor S. Klainerman for guidance and encouragement. Thanks are also due to Professor D. Christodoulou for many helpful suggestions. 


\section{References}

1. Choquet-Bruhat, Y., Christodoulou, D.: Existence of global solutions of the Yang-Mills, Higgs and spinor field equations in $3+1$ dimensions, Ann. Sci. Ecole Norm. Sup. 14, 481-506 (1981)

2. Christodoulou, D.: Global solutions of nonlinear hyperbolic equations for small initial data. Commun. Pure Appl. Math. 39 (2), 267-282 (1986)

3. Christodoulou, D., Klainerman, S.: Asymptotic behavior of linear field equations in Minkowski space. Commun. Pure Appl. Math. 1990

4. Christodoulou, D., Klainerman, S.: The global nonlinear stability of the Minkowski space (in press)

5. Eardley, D. M., Moncrief, V.: The global existence of Yang-Mills fields in 4-dimensional Minkowski space, Part 1: Local existence and smoothness properties. Commun. Math. Phys. 83, 171-191 (1982)

6. Eardley, D. M., Moncrief, V.: The global existence of Yang-Mills fields in 4-dimensional Minkowski space, Part 2: Completion of Proof. Commun. Math. Phys. 83, 193-212 (1982)

7. Ginbre, J. Velo, G.: The Cauchy Problem for coupled Yang-Mills and Scalar fields in Temporal Gauge. Commun. Math. Phys. 82, 1-28 (1982); on pairs of null directions. J. Math. Phys. 14 (7). 874-881 (1973)

8. Klainerman, S.: Uniform decay estimates and the Lorentz invariance of the classical wave equation. Commun. Pure Appl. Math. 38 (3), 321-332 (1985)

9. Klainerman, S.: The null condition and global existence to nonlinear wave equations. Lectures in Appl. Math. 23 (Part I), Providence, RI: Am. Math. Soc. 1986

10. Newman, E. T., Penrose, R.: An Approach to gravitational radiation by a method of spin coefficients. J. Math. Phys. 3, 896-902 (1962)

11. Penrose, R.: Zero rest-mass fields including gravitation: asymptotic behaviour. Proc. R. Soc. London A284, 159-203 (1965)

12. Penrose, R., Rindler, W.: Spinors and Space-time, Vol. 1, Two-spinors calculus and relativistic fields. Cambridge: Cambridge University Press 1986

13. Shu, W.: Spin Field Equations and Yang-Mills Equation, Ph.D. thesis, Princeton University

Communicated by T. Spencer 